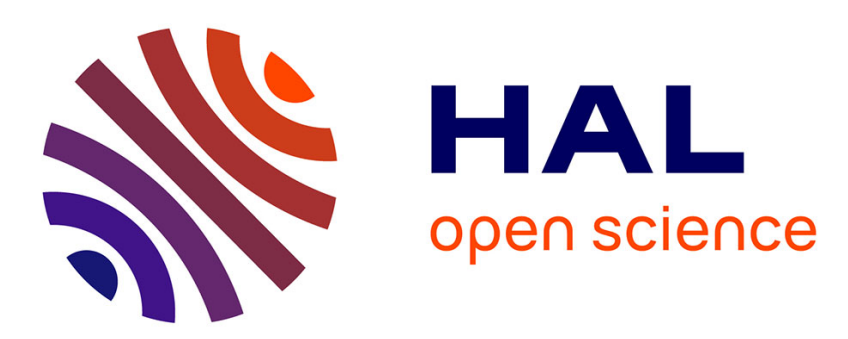

\title{
Bifurcation problems for Ginzburg-Landau equations and applications to Bose Einstein condensates
}

\author{
Amandine Aftalion
}

\section{To cite this version:}

Amandine Aftalion. Bifurcation problems for Ginzburg-Landau equations and applications to Bose Einstein condensates. 3rd cycle. Shanghaï (Chine), 2002, pp.51. cel-00376322

HAL Id: cel-00376322

https://cel.hal.science/cel-00376322

Submitted on 17 Apr 2009

HAL is a multi-disciplinary open access archive for the deposit and dissemination of scientific research documents, whether they are published or not. The documents may come from teaching and research institutions in France or abroad, or from public or private research centers.
L'archive ouverte pluridisciplinaire HAL, est destinée au dépôt et à la diffusion de documents scientifiques de niveau recherche, publiés ou non, émanant des établissements d'enseignement et de recherche français ou étrangers, des laboratoires publics ou privés. 
CIMPA SCHOOL: Ginzburg-Landau vortices

$$
\text { Shanghaï, } 2002 \text { November } 18^{t h}-29^{t h}
$$

\section{Mini-course:}

Bifurcation problems for Ginzburg-Landau equations and applications to

Bose Einstein condensates

\section{Amandine AFTALION CNRS and University Paris 6}




\section{Introduction}

This course is dedicated to the study of bifurcation diagrams in the framework of the Ginzburg-Landau theory. The aim is to characterize the properties of solutions according to the values of the different free parameters: existence of non trivial solutions, number of solutions, stability and symmetry.

In the first two chapters, we study the case of a superconducting material placed in a magnetic field for two different geometries. In the Ginzburg-Landau model, the state of the system can be described by a system of two coupled PDE's. In chapter 1, for the infinite slab, the equations get scalar and reduce to a system of two coupled ODE's. Chapter 2 deals with the infinite cylinder, which is a $2 \mathrm{~d}$ problem.

The 3rd chapter addresses a related problem: vortices in rotating Bose Einstein condensates. The physical phenomenon is modeled by a nonlinear Schrodinger equation with a trapping and rotating term. Analogies are made with Ginzburg-Landau and symmetry and breaking of symmetry of $3 \mathrm{~d}$ vortices are analyzed. 


\section{Chapter 1}

\section{The Ginzburg-Landau system in dimension 1}

\subsection{Introduction}

The superconductivity of certain metals is characterized at very low temperatures by the loss of electrical resistance and the expulsion of the exterior magnetic field $\mathbf{h}_{0}$. In the model derived by Ginzburg and Landau in 1950 (see [GL]), the electromagnetic properties of the material are completely described by the magnetic potential vector $\mathbf{A}(\mathbf{h}=\operatorname{curl} \mathbf{A}$ being the magnetic field) and the complex-valued order parameter $\psi$. In fact, $\psi$ is an averaged wave function of the superconducting electrons and its modulus corresponds to the density of superconducting carriers. When the sample is wholly normal, $|\psi| \equiv 0$ and the magnetic field inside the material $\mathbf{h}$ is equal to the exterior magnetic field $\mathbf{h}_{0}$. On the other hand, when the sample is perfectly superconducting, $|\psi| \equiv 1$ and the magnetic field $\mathbf{h}$ is identically 0. Furthermore, in the Ginzburg-Landau theory, the state of the sample is completely determined by the minimum of an energy depending on $\psi$ and $\mathbf{A}$. For a more precise description of the general theory, one may refer to $[\mathrm{CP}],[\mathrm{G}],[\mathrm{GL}]$, [SJdG], [TT], $[\mathrm{T}]$ or to [CHO], [DGP1].

In the special case when the sample is an infinite slab of constant thickness, between the planes $x=-a$ and $x=a$, it is usual to assume that both $\psi$ and $\mathbf{A}$ are uniform in the $y$ and $z$ directions, and that the exterior magnetic field is tangential to the slab, that is $\mathbf{h}_{0}=\left(0,0, h_{0}\right)$. A suitable gauge can then be chosen so that $\psi=f(x)$ is a real function, and $\mathbf{A}=q(x) \mathbf{e}_{y}$, where $\mathbf{e}_{y}$ is the unit vector along the $y$ direction (see [GL] for more details). In this case, the nondimensionalized form of the Ginzburg-Landau energy is given by:

$$
E_{\kappa}(f, q)=\int_{-a}^{a}\left(\frac{1}{\kappa^{2}} f^{\prime 2}+f^{2} q^{2}+\frac{1}{2} f^{4}-f^{2}+\left(q^{\prime}-h_{0}\right)^{2}\right) d x
$$

The nondimensionalized parameter $\kappa$ is called the Ginzburg-Landau parameter. It is the ratio of $\lambda$, the penetration depth of the magnetic field, to $\xi$, the coherence length, which is the characteristic length of variation of $f$. The value of $\kappa$ determines the type of superconductor according to the type of phase transition which takes place between the normal 
phase and the superconducting phase: $\kappa$ small describes what is known as a type I superconductor and $\kappa$ large as a type II. More precisely, for a type I superconductor, there is a critical magnetic field $h_{c}$ such that if $h_{0}<h_{c}$, the material is entirely superconducting and the magnetic field is expelled from the sample apart from a boundary layer of size $\lambda$. This is called the Meissner effect. If $h_{0}>h_{c}$, superconductivity is destroyed and the material is in the normal state, that is $f \equiv 0$ and $q^{\prime} \equiv h_{0}$. For a type II superconductor, the phase transition is different and there are two critical fields $h_{c_{1}}$ and $h_{c_{2}}$ : for $h_{0}<h_{c_{1}}$, the exterior magnetic field is expelled from the sample and there is a Meissner effect as for type I superconductors. But as $h_{0}$ is increased above $h_{c_{1}}$, superconductivity is not destroyed straight away, since the superconducting and the normal phase coexist under the form of filaments or vortices: the vortex is a zone of diameter $\xi$, at the center of which the order parameter $f$ vanishes. As $h_{0}$ increases further, the vortices become more numerous until the critical value $h_{c_{2}}$ is reached at which superconductivity is destroyed. For $h_{0}>h_{c_{2}}$, there is no superconductivity and the material is in the normal state. The way superconductivity is nucleated is highly dependent on $a$ and $\kappa$. We refer to Tinkham [T] for a detailed explanation. The vortex phenomena in superconductivity have been widely studied in the literature. See for instance $[\mathrm{BBH}]$ or [DGP1] and the references therein. The critical value of $\kappa$ usually given to separate type I and type II superconductors is $\kappa=1 / \sqrt{2}$. We will describe how this value is computed in the limiting case $a=\infty$. However, as $a$ is decreased from infinity, we will see that the demarcation between type I and II behaviours is no longer the constant $\kappa=1 / \sqrt{2}$. Instead, we find that there is a well defined curve in the $(\kappa, a)$ plane which separates the two types of behaviours.

For a mathematical analysis of the problem, it is natural to assume that $f \in H^{1}(-a, a)$ and $q \in H^{1}(-a, a)$. It then follows from standard variational arguments that there exists a minimizer of $E_{\kappa}$, and that the minimizer is a solution of

$$
\left\{\begin{array}{l}
\frac{1}{\kappa^{2}} f^{\prime \prime}=f\left(f^{2}+q^{2}-1\right) \quad \text { in }(-a, a), \quad f^{\prime}( \pm a)=0 \\
q^{\prime \prime}=q f^{2} \quad \text { in }(-a, a), \quad q^{\prime}( \pm a)=h_{0} .
\end{array}\right.
$$

Notice that $f \equiv 0$ and $q(x)=h_{0}(x+e)$ is always a solution for any real $e$. From now on, we will call this a normal solution. Regularity properties of minimizers yield that either $f$ is a normal solution, or $f$ does not change sign, hence we will study the case $f>0$. An easy calculation shows that the energy $E_{\kappa}$ is zero along the normal solution. Thus a global minimizer cannot have positive energy.

We want to give a complete description (number, symmetry and stability) of the solutions of the system $(G L)$ for which $f>0$ on $[-a, a]$, according to the values of the parameters $a, \kappa$ and $h_{0}$. Let us first recall the basic properties of solutions.

Proposition 1.1 If $(f, q)$ is a solution of $(G L)$ and if $f$ is not identically zero then

(i) $|f| \leq 1$ in $(-a, a)$, 


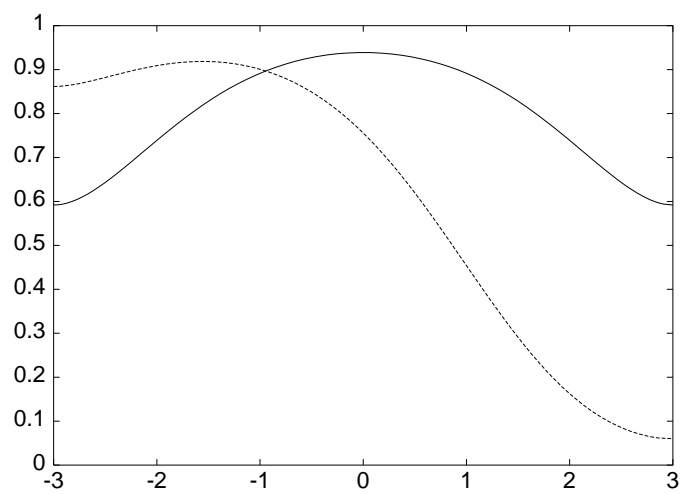

(a)

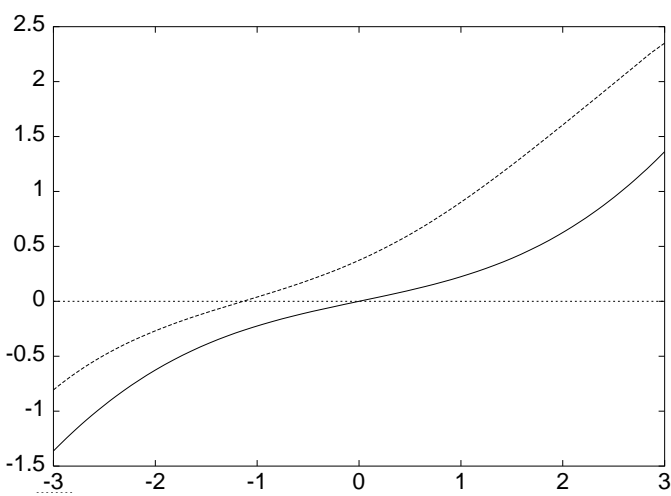

(b)

Figure 1.1: Symmetric solutions (full line) and asymmetric solutions (dotted line) (a) $f$ and (b) $q$, for $a=3, \kappa=0.9$.

(ii) $q$ has a unique zero $a_{0}$ in $(-a, a), q$ is increasing on $(-a, a), q^{\prime}$ is decreasing on $\left(-a, a_{0}\right)$ and increasing on $\left(a_{0}, a\right)$.

(iii) There exist $x_{1}$ and $x_{2}$ with $-a \leq x_{1} \leq a_{0} \leq x_{2} \leq a$ and $x_{0} \in\left[x_{1}, x_{2}\right]$ such that $f^{\prime}$ is increasing on $\left[-a, x_{1}\right] \cup\left[x_{2}, a\right]$ and decreasing on $\left[x_{1}, x_{2}\right], f$ is increasing on $\left[-a, x_{0}\right]$ and decreasing on $\left[x_{0}, a\right]$.

The proof of $(i)$ and $(i i)$ can be found for instance in [BH3] and of $(i i i)$ in [A1].

There are two types of physically important solutions of $(G L)$ : symmetric solutions and asymmetric solutions (see Figure 1.1). We define a symmetric solution to be a solution of $(G L)$ such that $f>0, f$ is even and $q$ is odd on $[-a, a]$. Thus, a symmetric solution satisfies the following problem:

$$
\left\{\begin{array}{l}
\frac{1}{\kappa^{2}} f^{\prime \prime}=f\left(f^{2}+q^{2}-1\right) \quad \text { in }(0, a), \quad f(0)=\beta, \quad f^{\prime}(0)=0 \\
q^{\prime \prime}=q f^{2} \quad \text { in }(0, a), \quad q(0)=0, \quad q^{\prime}(0)=\alpha,
\end{array}\right.
$$

for $\beta \in(0,1)$ and $\alpha \geq 0$. We need to choose $\alpha$ and $\beta$ such that $f^{\prime}(a)=0$. Then $(f, q)$ will be a solution of $(G L)$ with $h_{0}=q^{\prime}(a)$. Notice that $\beta$ is the amplitude of $f$ for a symmetric solution.

We define an asymmetric solution to be a solution of $(G L)$ which satisfies $f>0$ on $[-a, a]$, yet which is not symmetric. That is $f^{\prime}(0) \neq 0$ or $q(0) \neq 0$.

It is important to understand that symmetric solutions correspond to bulk superconductivity. When $h_{0}$ is decreased from infinity, superconductivity is not always nucleated first in the volume of the sample, which would give rise to symmetric solutions, but sometimes rather in a sheath near the surface, due to the existence of asymmetric solutions. 
This is called surface superconductivity. If the slab is very thick, the two surface solutions $f(x)$ and $f(-x)$ do not interact. In this region, superconductivity is first nucleated in surface layers of size $1 / \kappa$ near the boundaries, and the middle part of the material is normal. Now, if the slab is of intermediate size, the solutions $f(x)$ and $f(-x)$ interfere to create vortices. Indeed, the original Ginzburg-Landau energy is gauge invariant so that a solution $(f(x), q(x))$ has the same energy as $\left(e^{i \kappa c y} f(x), q(x)+c\right)$ for any constant $c$. Thus, when the sample is not too large, the linear combination of the asymmetric solutions $f(x)$ and $f(-x)$ create two dimensionnal vortices along the mid-plane $x=0$. This is reflected in the formula:

$$
\psi=\cos k y(f(x)+f(-x))+i \sin k y(f(x)-f(-x))
$$

Further discussion of vortex formation and the details of the derivation of formula (1.2) are given in Tinkham $[\mathrm{T}]$.

We are now going to study the existence and multiplicity of solutions.

\section{$1.2 \quad$ Symmetric solutions}

For the existence of symmetric solutions, Kwong $[\mathrm{Kw}]$ has proved the following important result.

Theorem 1.1 (Kwong $[K w]$ ) For each $\beta$ in $(0,1)$, there exists a unique $\alpha>0$ such that the solution $(f, q)$ of $\left(G L_{\text {sym }}\right)$ satisfies $f^{\prime}(a)=0$. Moreover, $\alpha$ is a continuous, decreasing function of $\beta$,

$$
\lim _{\beta \rightarrow 0} \alpha(\beta)>0 \quad \text { and } \quad \lim _{\beta \rightarrow 1} \alpha(\beta)=0 .
$$

For this choice of $\alpha(\beta)$, and the corresponding solution $(f, q)$ of $\left(G L_{\text {sym }}\right)$, let $h(\beta)=q^{\prime}(a)$. Then $h$ is well-defined, continuous and

$$
\lim _{\beta \rightarrow 0} h(\beta)=h_{s}>0 \quad \text { and } \quad \lim _{\beta \rightarrow 1} h(\beta)=0 .
$$

\subsubsection{Numerical results}

There are three possible behaviours of the curve $h(\beta)$ defined in Kwong's Theorem, and these are shown in Figures 1.2, 1.3 and 1.4. Notice that instead of graphing $h(\beta)$ vs $\beta$, we have put $\beta$ on the vertical axis and $h$ on the horizontal axis, so that we keep the convention originally adopted by Ginzburg [G, Figures 3 and 5]. This numerical simulations are obtained with AUTO, a software developed by Doedel et al. (see [Do1], [Do2]) which computes bifurcation diagrams for systems of ODE's.

- Figure 1.2: for $\beta \in(0,1), h(\beta)$ is a decreasing function of $\beta$ : if $0<h_{0}<h_{s}$, there is exactly one symmetric solution of $(G L)$, and if $h_{0} \geq h_{s}$, no such solution. 


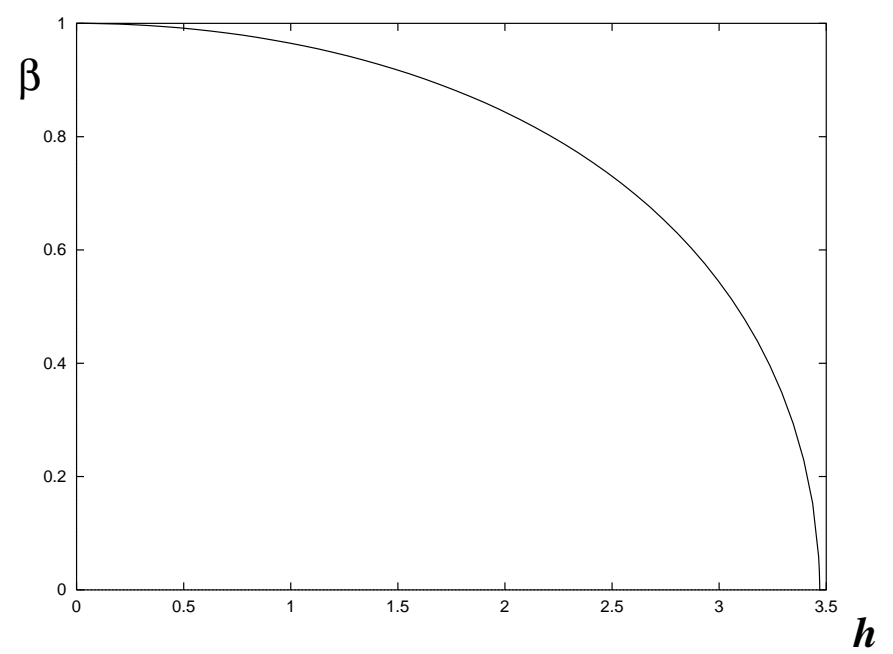

Figure 1.2: Curve $h(\beta)$ for $a=0.5$ and $\kappa=0.4$

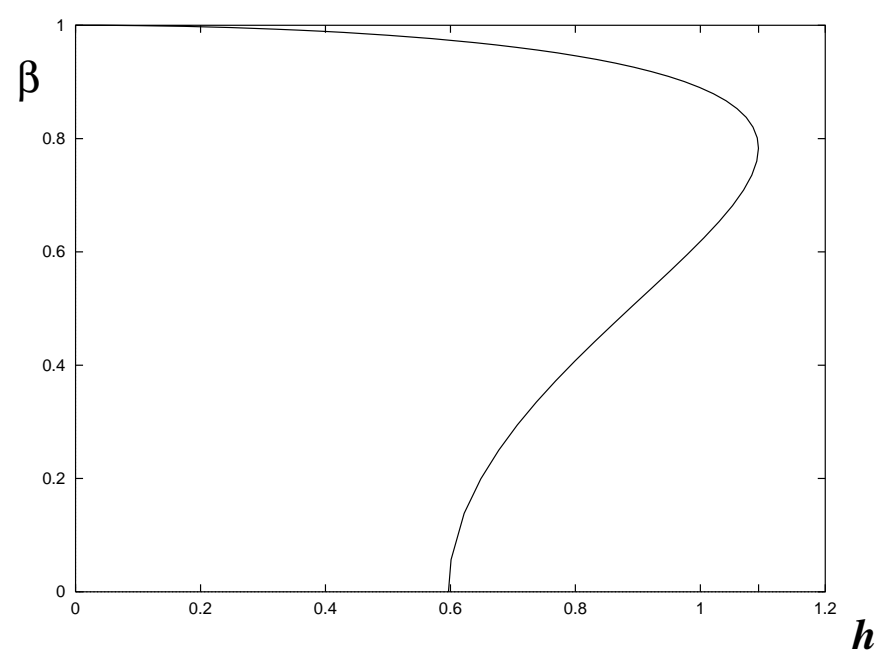

Figure 1.3: Curve $h(\beta)$ for $a=3$ and $\kappa=0.3$

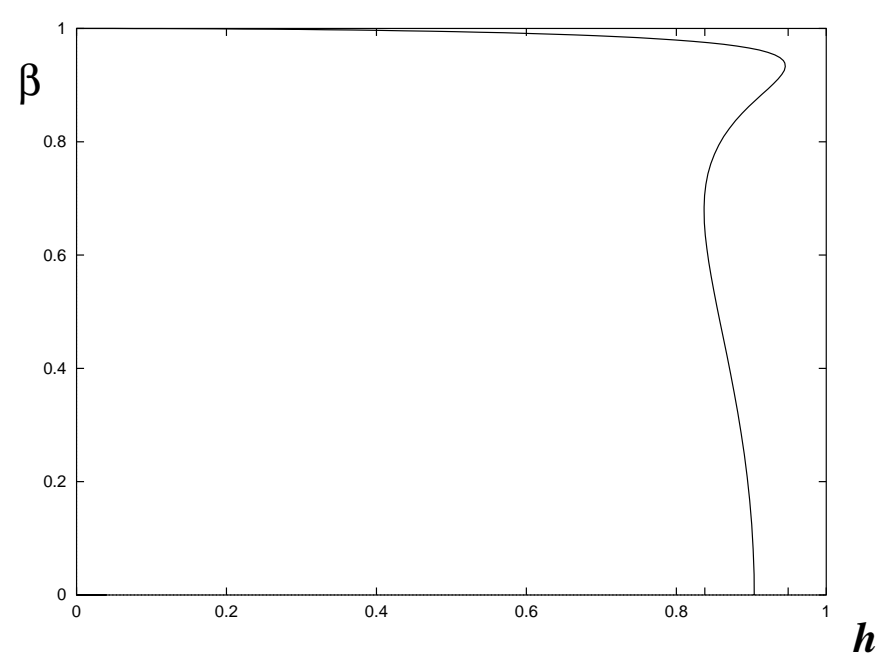

Figure 1.4: Curve $h(\beta)$ for $a=3$ and $\kappa=0.9$ 
- Figure 1.3: for $\beta \in(0,1), h(\beta)$ is increasing until $h$ reaches a maximum value $\bar{h}$ (here $\bar{h} \simeq 1.1$, and then decreasing to 0 as $\beta$ goes to 1 . Thus, for $h_{0} \leq h_{s},(G L)$ has a unique symmetric solution, for $h_{s}<h_{0}<\bar{h}$, two symmetric solutions, and for $h_{0}>\bar{h}$ no such solution.

- Figure 1.4: for $\beta \in(0,1), h(\beta)$ is decreasing until $h$ reaches a local minimum value $\underline{h}$ (here $\underline{h} \simeq 0.83$ ), increasing until $h$ reaches a local maximum value $\bar{h}$ (here $\bar{h} \simeq 0.95$ ), and then decreasing to 0 as $\beta$ goes to 1 . There $\underline{h}<h_{s}<\bar{h}$. Thus, for $h_{0}<\underline{h},(G L)$ has a unique symmetric solution, for $\underline{h}<h_{0}<h_{s}$, three symmetric solutions, for $h_{s} \leq h_{0}<\bar{h}$, two symmetric solutions, and for $h_{0}>\bar{h}$ no symmetric solution.

If $\bar{h}<h_{s}$, then for $h_{0}<\underline{h},(G L)$ has a unique symmetric solution, for $\underline{h}<h_{0}<\bar{h}$, three symmetric solutions, for $\bar{h}<h_{0}<h_{s}$, a unique symmetric solution, and for $h_{0} \geq h_{s}$ no symmetric solution.

Note that in Figure 1.3 and 1.4, there are points on the curve where the resultant slope is vertical, when $h=\underline{h}$ or $\bar{h}$. We shall refer to such points as folds.

Our simulations also indicate that the $(a, \kappa)$ plane is the union of three connected sets $S_{1}, S_{2}$ and $S_{3}$ as shown in Figure 1.5. In $S_{1}$, the behaviour of $h(\beta)$ of Figure 1.2 holds. Likewise, $S_{2}$ and $S_{3}$ reflect the behaviour of $h(\beta)$ of Figures 1.3 and 1.4 respectively. The region $S_{1}$ corresponds to thin films for which there is at most one solution. The passage from $S_{1}$ to $S_{2}$ was known for $\kappa$ small, and from $S_{2}$ to $S_{3}$ for $a$ large. The difference between $S_{2}$ and $S_{3}$ corresponds to going from type I to type II. The critical value is $1 / \sqrt{2}$ for $a$ large but depends on $a$.

\subsubsection{Rigorous results}

The bifurcation diagrams indicate that if the applied field $h_{0}$ is sufficiently strong, superconductivity is destroyed. This has been proved by Kwong $[\mathrm{Kw}]$.

Proposition 1.2 (Kwong $[K w]$ ) For any $a$ and $\kappa$, there exists $h_{c}$ such that for $h_{0}>h_{c}$, the only solution of $(G L)$ is the normal solution $f \equiv 0, q^{\prime} \equiv h_{0}$.

As each of the Figures indicates, as one decreases $h_{0}$ from infinity, the material remains in the normal state until a critical value of $h_{0}$ is reached at which there is a bifurcation of nontrivial solutions from the normal state.

So for small $\varepsilon>0$, a nontrivial curve $(f(., \varepsilon), q(., \varepsilon), h(\varepsilon))$ of solutions of $(G L)$ starting from a normal solution $\left(0, h_{0}(x+e), h_{0}\right)$ is sought, with the following asymptotic development:

$$
\begin{aligned}
& f(x, \varepsilon)=\varepsilon f_{0}(x)+\varepsilon^{3} f_{1}(x)+o\left(\varepsilon^{3}\right) \text { in } H^{2}(-a, a), \\
& q(x, \varepsilon)=q_{0}(x)+\varepsilon^{2} q_{1}(x)+o\left(\varepsilon^{2}\right) \text { in } H^{2}(-a, a), \\
& h(\varepsilon)=h_{0}+\varepsilon^{2} h_{1}+\varepsilon^{4} h_{2}+o\left(\varepsilon^{4}\right),
\end{aligned}
$$

where $q_{0}(x)=h_{0}(x+e)$. It is important to note that when $e=0$, the branch gives rise to symmetric solutions and when $e \neq 0$ to asymmetric solutions. At first order, this leads to 


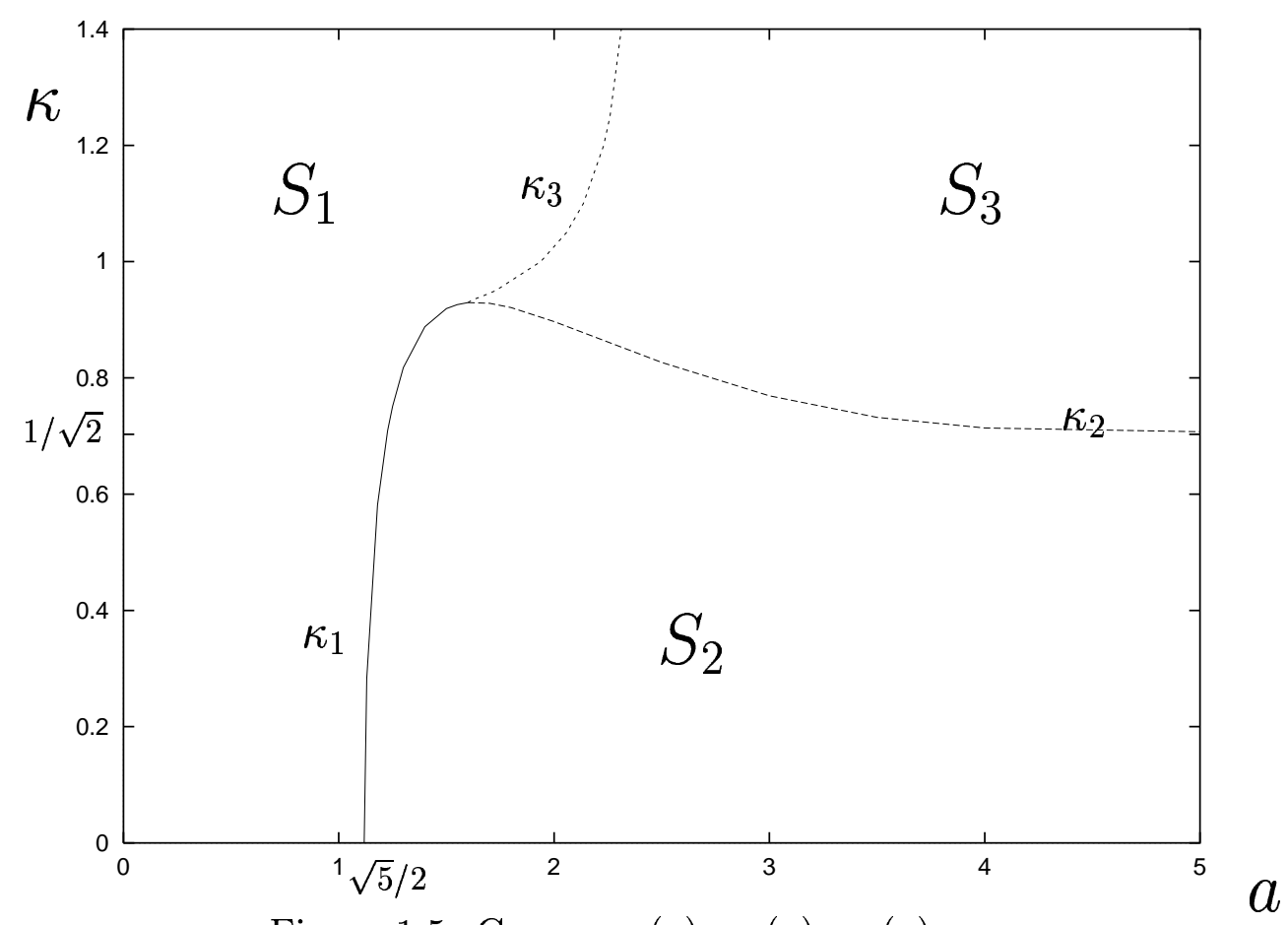

Figure 1.5: Curves $\kappa_{1}(a), \kappa_{2}(a), \kappa_{3}(a)$.

an eigenvalue problem

$$
\frac{1}{\kappa^{2}} f_{0}^{\prime \prime}=f_{0}\left(h_{0} x-1\right), \quad f_{0}^{\prime}( \pm a)=0 .
$$

Furthermore, it can be proved (see [Ch1] or [BH2] for more details) that for small $\varepsilon>0$, the energy of the bifurcated superconducting solution, $E_{\kappa}(f(., \varepsilon), q(., \varepsilon))$, has the same sign as $h_{1}$. A curve of the form (1.5) is said to result from a supercritical bifurcation if $h_{1}<0$. Then, for small $\varepsilon>0$, the bifurcated solutions have lower energy than the normal state. A bifurcation is said to be subcritical if $h_{1}>0$. Here the bifurcated solutions have larger energy than the normal state. This means that for Figures 1.2 and 1.4, the bifurcation is supercritical and for Figure 1.3 subcritical.

We now summarize the main results previously obtained concerning these figures describing symmetric solutions. We note that Ginzburg $[\mathrm{G}]$ had investigated the case $\kappa$ small and found that $h(\beta)$ behaves as in Figure 1.2 for small $a$ and, as $a$ is increased through a critical value, the graph of $h(\beta)$ changes from Figure 1.2 to 1.3. He explained the type of behaviour described by Figure 1.3 in terms of superheating and supercooling. More precisely, when $h_{0}$ is large, superconductivity does not occur and the material is in the normal state. As $h_{0}$ is decreased, the material stays in the normal state down to $h_{s}$, even though there is a range of $h_{0}$ where the normal solution is only a local minimizer and the global minimizer is a superconducting solution (see [G, Figure 2]). If $h_{0}$ is decreased further, there is a jump in the maximum of $f$ and the material becomes superconducting, the solution being given by the symmetric branch. In this case, $h_{s}$ is called the supercooling field. Now, on the contrary, start from $h_{0}=0$ where the superconducting state $(1,0)$ is 
the global minimizer and increase $h_{0}$. The material will remain superconducting until $\bar{h}$ is reached, though for fields slightly less then $\bar{h}$, it is only a local minimizer and the global minimizer is the normal solution. For $h_{0}$ above $\bar{h}$, there is a jump in the maximum of $f$ and the material reverts to the normal state. This is the superheating phenomenon. These two phenomena give rise to a hysteresis loop as described in [G] or later in [DGP1].

Ginzburg and Landau [GL] had also noticed that if $a$ large is fixed, then there occurs a symmetric supercritical bifurcation of superconducting solutions from the normal state as $\kappa$ is increased through a critical value, but had no special explanation for this, since at that time only superconductors with small $\kappa$ were known. However, as $\kappa$ is increased through this critical value, our studies indicate that the behaviour seen in Figure 1.3 changes into that seen in Figure 1.4.

Chapman [Ch1] has studied the case $a=\infty$, and showed that there is a change of bifurcation from subcritical to supercritical that takes place for $\kappa=1 / \sqrt{2}$, which is the critical value between type I and II superconductors. Moreover, in [Ch2], a linear stability analysis through the time dependent equations yield that the value $\kappa=1 / \sqrt{2}$ is also the one for which stability of the normal solution switches.

Following these works which are mainly based on formal computations, Bolley and Helffer have extensively studied the phenomenon of bifurcation of solutions from the normal solution in [BH1], [BH2], [BH3], [BH4], [BH5], [BH6]. In particular, they have given rigorous proofs of properties of bifurcating branches and asymptotic formula for the superheating and supercooling fields. Although their results are mainly local, they have made a first attempt in [BH6] to give a global stability picture of the solutions.

Another type of study of these bifurcation diagrams consists in trying to get global properties, not only near the normal solution. Hastings, Kwong and Troy [HKT] have further investigated the multiplicity of symmetric solutions. They have proved that if $\kappa \leq 1 / \sqrt{2}$, and $a$ is sufficiently large, then the behaviour of $h(\beta)$ is described by Figure 1.3 , that is there is a region of $h_{0}$ for which there are at least 2 solutions, and if $\kappa>1 / \sqrt{2}$ and $a$ sufficiently large, by Figure 1.4, that is there is a region of $h_{0}$ for which there are at least 3 solutions.

Another type of results deals with $S_{1}$.

Theorem 1.2 For each $\kappa>0$ there exists $\bar{a}=\bar{a}(\kappa)$ such that for $0<a<\bar{a}(\kappa)$, the curve $h(\beta)$ defined in Theorem 1.1 is decreasing for $0<\beta<1$ and there is no bifurcation of asymmetric solutions from the symmetric branch. Moreover, there exist positive constants $a_{0}$ and $a_{1}$ independent of $\kappa$ and $\beta$, such that $\kappa \bar{a}(\kappa) \leq a_{0}$ and $\bar{a}(\kappa) \leq a_{1}$.

Corollary 1.1 Let $\kappa>0$ and $0<a<\bar{a}(\kappa)$. For each $h_{0}$ in $\left(0, h_{s}\right)$ there exists a unique symmetric solution of $(G L)$. If $h_{0} \geq h_{s}$, there is no symmetric solution.

\subsection{Asymmetric solutions}

As we have said earlier, asymmetric solutions correspond to vortices in this geometry. Below, we summarize the results of our numerical investigation of asymmetric solutions 
shown in Figures 1.6 and 1.7: the vertical axis has been labeled $\|f\|$. For the symmetric branch, $\|f\|$ refers to $f(0)=\beta$ as in Figures 1.2, 1.3 and 1.4. For the asymmetric branch, $\|f\|=\|f\|_{\infty}$, which is attained at some point $x_{\beta}$ different from zero. For simplicity, we will sometimes refer to the complete bifurcation diagram as $h(\beta)$. In Figures 1.6 and 1.7 , the curve $h(\beta)$ for symmetric solutions is the typical curve in $S_{2}$. On these curves, there is a point $\left(\beta_{b}, h_{b}\right)$ denoted by a square: it is the branching point of the branch of asymmetric solutions. More precisely, for $\|f\|_{\infty}$ close to $\beta_{b}$, the asymmetric solution is nearly symmetric. The branch of asymmetric solutions leads from the branch of symmetric solutions to the branch of normal solutions. If the branch of asymmetric solutions crosses the branch of symmetric solutions without any square indicated, it just means that for this special $h_{0},(G L)$ has a symmetric solution and an asymmetric solution having the same maximum value of $f$.

We now describe the two possible behaviours of the asymmetric curve $h(\beta)$.

- Figure 1.6: for $\beta \in\left(0, \beta_{b}\right), h(\beta)$ is an increasing function of $\beta$ and $\lim _{\beta \rightarrow 0} h(\beta)=h_{a s}$, $\lim _{\beta \rightarrow \beta_{b}} h(\beta)=h_{b}$, with $h_{s}<h_{a s}<h_{b} \leq \bar{h}$. If $h_{0} \in\left(h_{a s}, h_{b}\right)$, there is a unique asymmetric solution of $(G L)$ and if $h_{0} \leq h_{a s}$ or $h_{0} \geq h_{b}$, no such solution.

- Figure 1.7: for $\beta \in\left(0, \beta_{b}\right), h(\beta)$ is a decreasing function of $\beta$ until $h$ reaches a minimum value $\underline{h}_{a s}$ and then increasing to $h_{b}$ as $\beta$ goes to $\beta_{b}$. thus, for $h_{0} \in\left(\underline{h}_{a s}, h_{b}\right)$, there are two asymmetric solution of $(G L)$, for $h_{0} \in\left(h_{b}, h_{a s}\right)$, a unique asymmetric solution and for $h_{0}<\underline{h}_{a s}$ or $h_{0} \geq h_{b}$, no such solution.

This implies in particular that for Figure 1.6 the bifurcation of asymmetric solutions from the normal solution is subcritical and for Figure 1.7 supercritical.

In Figure 1.8, we have determined the regions in the $(a, \kappa)$ plane where there is no asymmetric solution (region $A_{0}$ ), at most one pair of asymmetric solutions (region $A_{1}$ ) and at most two pairs of asymmetric solutions (region $A_{2}$ ). The curve $\kappa_{4}$ is defined by $a \kappa=c$.

Hastings and Troy have proved that for $\kappa>\kappa_{0}$ and $a$ large enough, then there is a range of $h_{0}$ for which there is no symmetric solution and yet there is an asymmetric solution. It means that $h_{a s}$ is much larger than the superheating field for symmetric solutions. In the region $A_{2}$, asymmetric solutions exist and are stable, which corresponds to surface superconductivity.

Let $\kappa$ be fixed. When $a$ tends to $\infty$, the bifurcated field of asymmetric solutions $h_{a s}$ tends to $\kappa / \mu_{1}^{0}$, where $\mu_{1}^{0}$ is approximately 0.59 , and is defined as the minimum over $\alpha$ of the first eigenvalue of the Neumann problem of the harmonic oscillator in $(-\alpha, \infty)[\mathrm{BH} 1]$. The curve $\kappa_{5}(a)$ corresponds to the solution when the asymmetric bifurcated curve changes stability. This happens when $h_{a s}$ is equal to $1 / \sqrt{2}$, hence the limit of $\kappa_{5}(a)$ for $a$ large is $\kappa_{a s}=\mu_{1}^{0} / \sqrt{2}$.

In $[\mathrm{AC} 1, \mathrm{AC} 2]$, the bifurcated solutions are studied near the curves $\kappa_{i}$. It turns out that the amplitude of the solution is not determined at first order, but fixed by a solvability condition at higher order. For most values of $a$ and $\kappa$, this is at first order, but along $\kappa_{i}(a)$, it is at even higher order. 


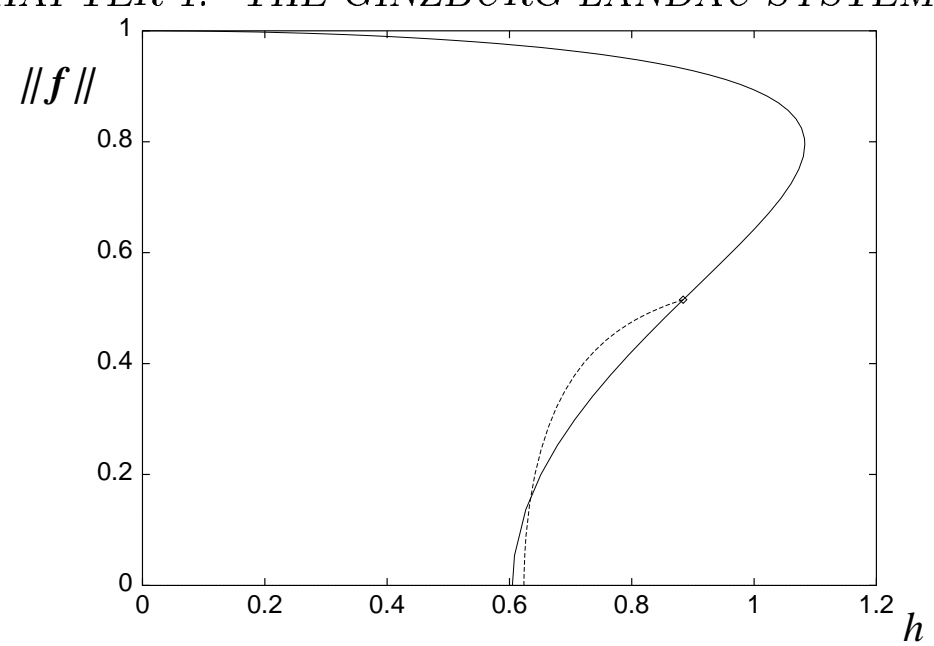

Figure 1.6: Bifurcation diagram for $a=3$ and $\kappa=0.35$.

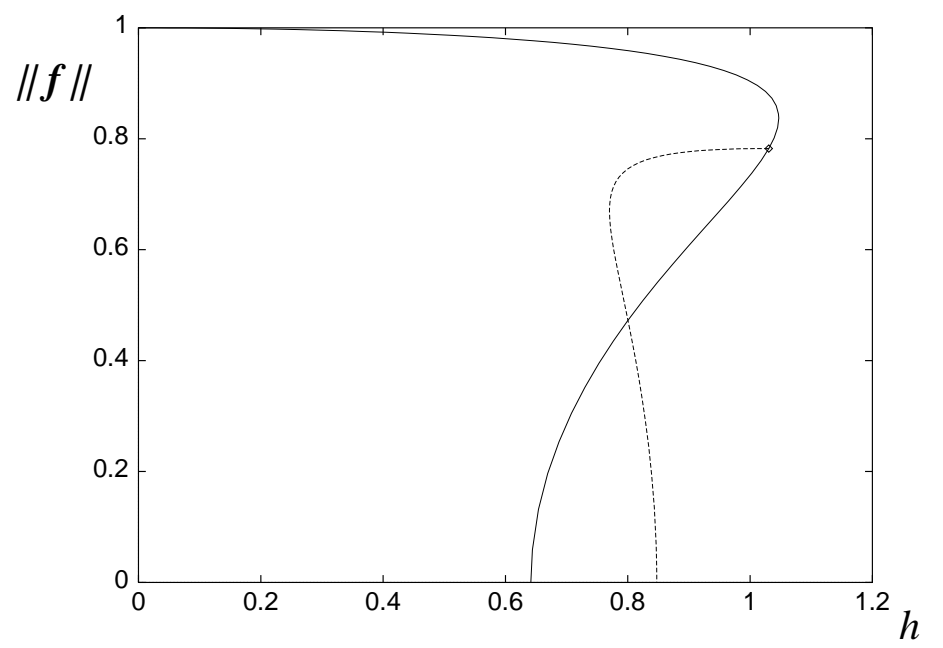

Figure 1.7: Bifurcation diagram for $a=3$ and $\kappa=0.5$.

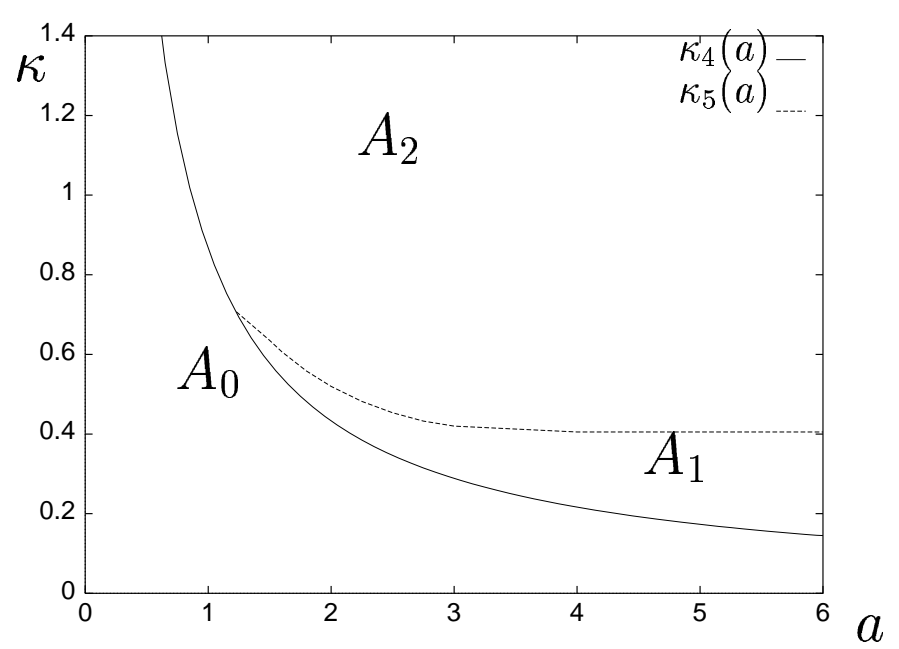

Figure 1.8: Curves $\kappa_{4}(a)$ and $\kappa_{5}(a)$. 


\section{Chapter 2}

\section{The Ginzburg-Landau model in dimension 2}

\section{$2.1 \quad$ Introduction}

In this part, we want to give a detailed description of the bifurcation diagrams of an infinite superconducting cylinder of cross section $\Omega$, submitted to an exterior magnetic field $h_{0}$. As in the previous section, the response of the material varies greatly according to the value of $h_{0}$, the size of the cross section and the Ginzburg-Landau parameter $\kappa$ that characterizes the material: superconductivity appears in the volume of the sample for low fields and small samples, under the form of vortices for higher fields, bigger samples and larger values of $\kappa$, and is destroyed for high fields. The type of response of a superconducting material has been studied numerically and theoretically by various authors in various asymptotic regimes [AD, BPT, BS, CDG, DFS, DPS, DGP1, DGP, GP, LP1, LP2, LP3, P, SS1, Ser1, Ser2, SP, SPD]. Here, we want to give a complete picture of the bifurcation diagrams for all values of the parameters.

$$
\mathcal{G}(\psi, A)=\int_{\Omega}|(\nabla-i A) \psi|^{2}+\frac{\kappa^{2}}{2}\left(1-|\psi|^{2}\right)^{2}+\left|\operatorname{curl} A-h_{0}\right|^{2} d \Omega
$$

where $\kappa$ is the Ginzburg-Landau parameter representing the ratio of the penetration depth and the coherence length, $h_{0}$ the applied magnetic field and $d$ the characteristic size of the domain $\Omega$, that is $\Omega=d D$ where $D$ is a fixed domain.

The system that we are going to study is the following Ginzburg-Landau equations derived as the Euler-Lagrange equations for the minimizers of the functional $\mathcal{G}$ [GL]:

$$
\left\{\begin{array}{l}
-(\nabla-i A)^{2} \psi=\kappa^{2} \psi\left(1-|\psi|^{2}\right) \text { in } \Omega, \\
-\operatorname{curl} \operatorname{curl} A=|\psi|^{2} A+\frac{i}{2}\left(\psi^{*} \nabla \psi-\psi \nabla \psi^{*}\right) \text { in } \Omega
\end{array}\right.
$$

which are supplemented by the boundary conditions

$$
\left\{\begin{array}{l}
(\nabla \psi-i A \psi) \cdot \mathrm{n}=0 \text { on } \partial \Omega \\
\operatorname{curl} A=h_{0} \text { on } \partial \Omega
\end{array}\right.
$$


and gauge constraints

$$
\left\{\begin{array}{l}
\operatorname{div} A=0 \text { in } \Omega \\
A \cdot \mathrm{n}=0 \text { on } \partial \Omega
\end{array}\right.
$$

Here, $\partial \Omega$ is the boundary of $\Omega$ and $\mathrm{n}$ is its unit outer normal. With the above nondimensionalization, $|\psi|$ takes values between 0 and 1: the normal state corresponds to $|\psi|=0$ while the Meissner state corresponds to $|\psi|=1$.

This Ginzburg-Landau model has a special family of solutions called the normal solutions: $\psi=0$ and curl $A=h_{0}$, which correspond to the situation where superconductivity is destroyed. According to the values of the different parameters $\kappa, d$ and $h_{0}$, the system may have other solutions: superconducting solutions, for which $\psi$ is never 0 and vortex solutions for which $\psi$ has isolated zeroes (see Figure 2.1 and 2.2). For a complete introduction to the topic, one may refer to $[\mathrm{T}]$. In Figure 2.1, we have a solution with a single vortex at the center of the domain which corresponds to the parameter values $\kappa=0.23$, $d=16.8$ and $h_{0}=0.563$. We have plotted respectively $|\psi|,|\operatorname{curl} A|$ and the current.
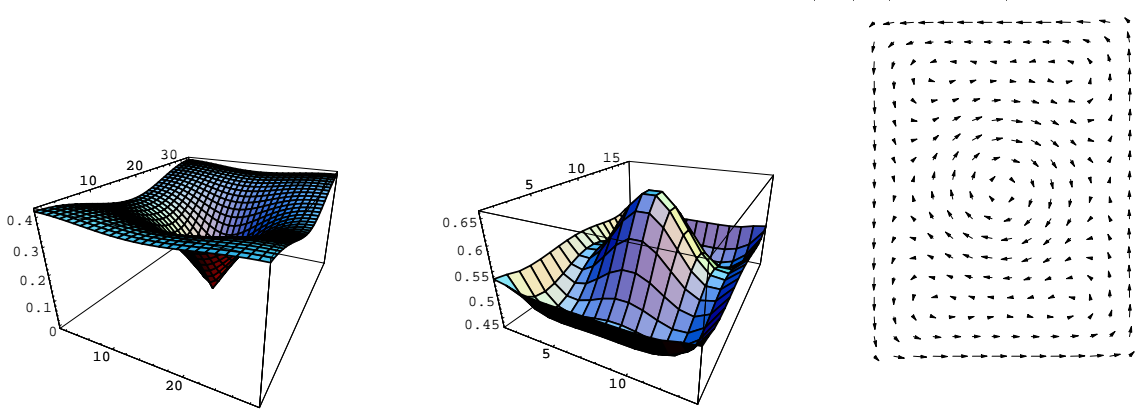

Figure 2.1: A single vortex solution of (2.1).

In Figure 2.2, we present the plots for a solution with two vortices corresponding to $\kappa=0.8, d=4$ and $h_{0}=1.2$.
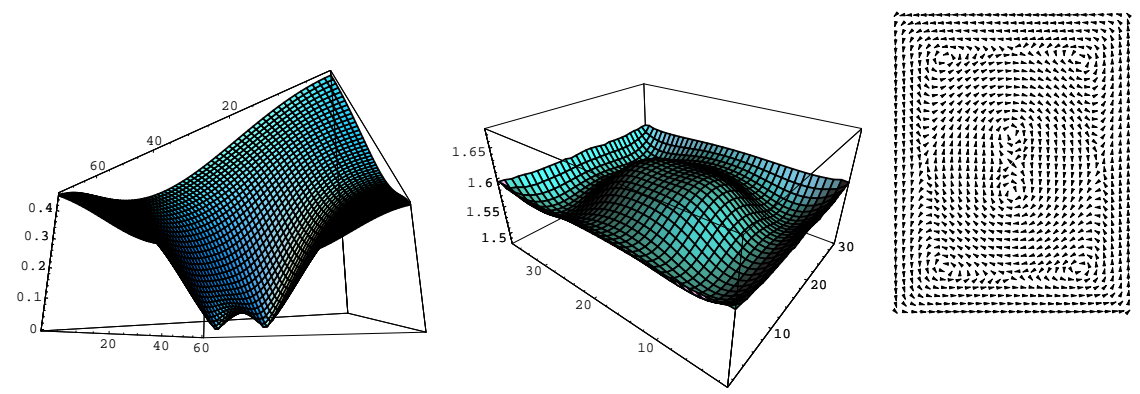

Figure 2.2: A solution of (2.1) with two vortices.

Here, the computation is made in a 2 dimensional domain using numerical solutions based on a code first developed in [DGP1]. 


\subsection{The bifurcation diagrams}

For fixed $\kappa, d$ and $h_{0}$, we are interested in finding the number of solutions of $(2.1)$ and their stability. A continuation in the parameter spaces is used for getting solutions with different parameter values. With $\kappa, d$ given but $h_{0}$ allowed to vary, for a computed solution branch, we plot $\|\psi\|_{\infty}$, the maximum magnitude of the order parameter $\psi$ in the domain and the free energy $\mathcal{G}$ versus the applied field $h_{0}$. These phase diagrams or bifurcation diagrams will give us information on the solutions (number and stability) for each $h_{0}$. The results of our numerical computations allow us to separate the $\kappa$-d plane into different regions depending on the shape of the bifurcation diagram.

It is well known that for large fields $\left(h>h_{*}\right)$, the only solution is the normal solution [GP]. For smaller fields $\left(h<h_{*}\right)$, the normal solution always exists but there are other solutions which display four different types of behaviors. These behaviors depend on the values of $\kappa$ and $d$. In Figure 2.3, we have plotted four curve segments $\left\{\kappa_{i}(d)\right\}_{1}^{4}$ separating the $\kappa-d$ plane into four regions $\left\{R_{i}\right\}_{1}^{4}$.

$\kappa$

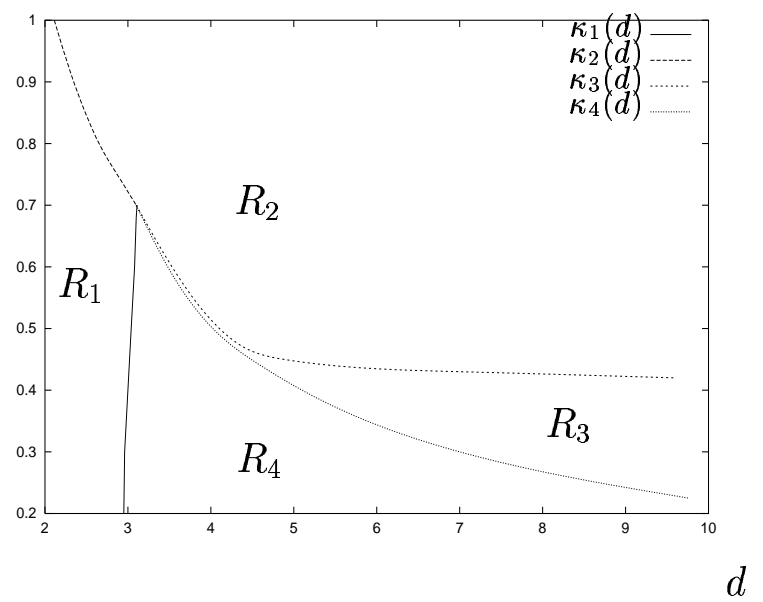

Figure 2.3: The curves $\kappa_{1}(d), \kappa_{2}(d), \kappa_{3}(d)$ and $\kappa_{4}(d)$

All four curves meet close to $\kappa=1 / \sqrt{2}, \kappa_{2}(d)$ is of the form $2.112 / d, \kappa_{3}(d)$ is tending to 0.4 at infinity. For convenience, for each $i=1,2,3,4$, we use $d=d_{i}(\kappa)$ to denote the inverse function of the function $\kappa=\kappa_{i}(d)$ wherever the inverse is well-defined. What distinguishes the different regions are features like the existence (or the lack of existence) of vortex solutions, the global and local stability of solutions, and the hysteresis phenomena. behavior for each region in Figure 2.3.

Region 1: $d<d_{1}(\kappa)$ and $d<d_{2}(\kappa)$.

This corresponds to the situation where the cross section of the superconducting sample is small enough. The bifurcation diagram is illustrated in Figure 2.4. The corresponding plot of the energy is given in Figure 2.5.

Throughout this region, there is a unique non-normal solution for $h<h_{*}$. This solution is a superconducting solution which is the global minimizer of the free energy $\mathcal{G}$. The curve 


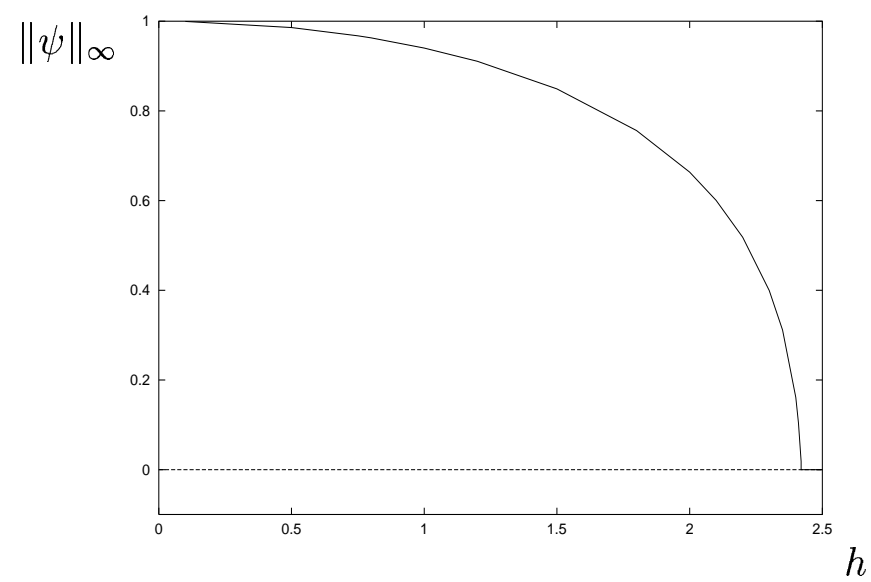

Figure 2.4: The bifurcation curve for $d=2.0 \kappa=0.3$

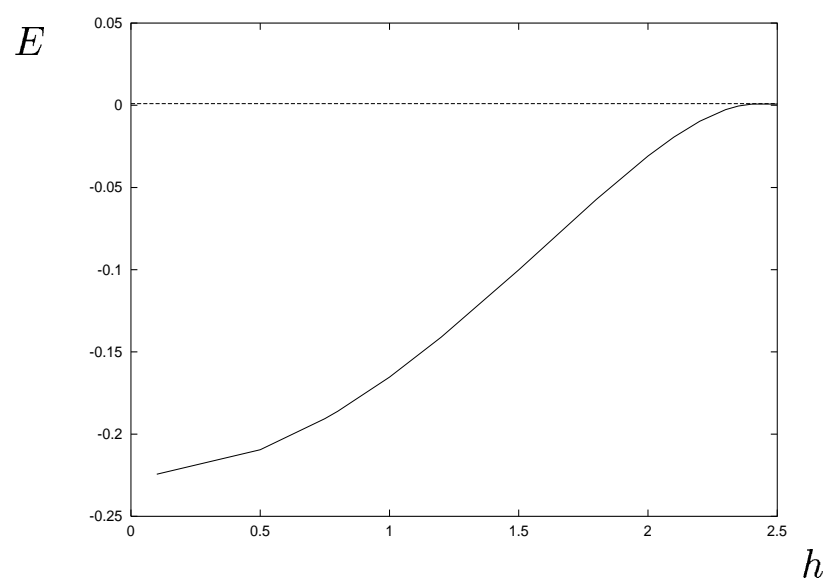

Figure 2.5: The energy for $d=2.0 \kappa=0.3$

$|\psi|_{\infty}$ against $h$ is monotonically decreasing. When increasing the field, the magnitude of the superconducting solution decreases until it turns normal at $h_{0}=h_{*}$. Conversely, when decreasing the field, the normal solution turns superconducting also for $h_{0}=h_{*}$. The transition to the normal solution is of second order, that is the energy of the superconducting solution tends to the energy of the normal solution at the transition and there is no hysteresis phenomenon.

There is no vortex solution for the parameters $(d, \kappa)$ in this region. This reflects the fact that $d$ is too small to allow enough room for a vortex to exist since a vortex core is of typical size $C / \kappa$. Rigorous results $[\mathrm{AD}]$ are given later.

Region 2: $\kappa>\kappa_{2}(d)$ and $\kappa>\kappa_{3}(d)$.

In contrast with Region 1, this region corresponds to the situation where the typical size of vortices $(C / \kappa)$ is small enough compared to the size of the domain. This region displays the typical type II behavior of superconductors. The bifurcation diagram is illustrated in 
Figure 2.6, the energy in Figure 2.7.

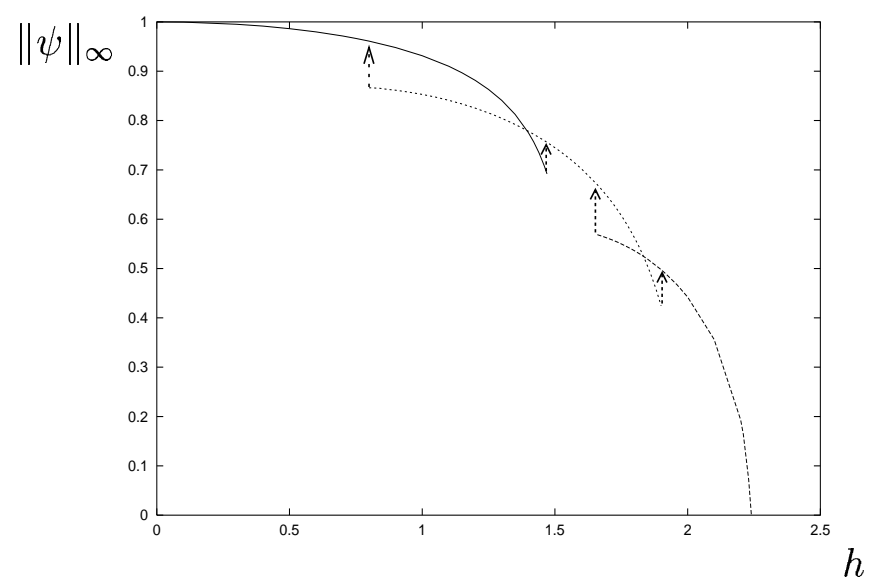

Figure 2.6: The bifurcation curve for $d=3.2 \kappa=1$

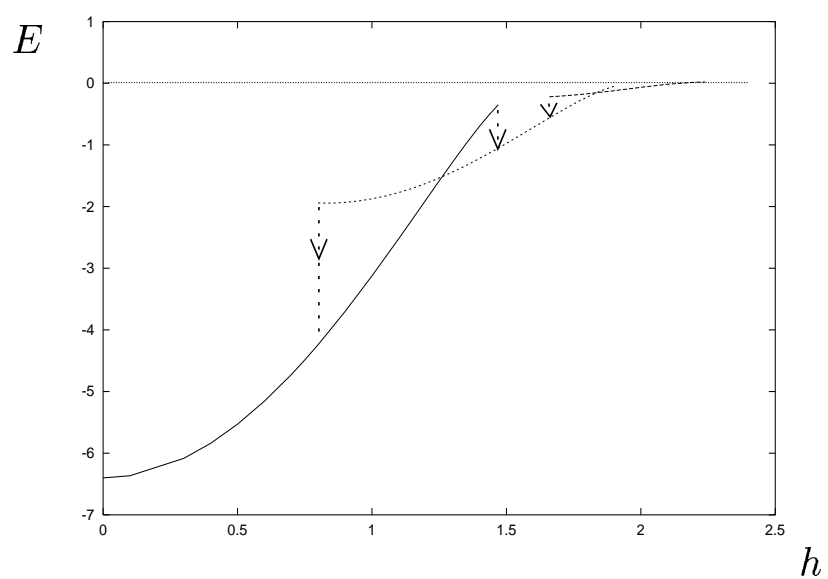

Figure 2.7: The energy for $d=3.2 \kappa=1$

It has been well understood both physically and in more recent years mathematically that, for sufficiently large $\kappa$, there are vortex solutions which are the global minimizers of the free energy for a certain range of fields. The number of vortices depends on the strength of the applied field. The maximum number of vortices increases with $d$ and $\kappa$.

For very small fields, the global minimizer is the superconducting solution (solid line). As the field is increased, the superconducting solution loses its global stability $\left(h=h_{c_{1}}\right.$ and for even larger fields loses its local stability, $h=h_{0}^{*}$ ). Then the global minimizer starts to nucleate vortices. In Figures 2.6, 2.7, the solution branch corresponding to a one vortex solution is illustrated by a dotted line and to a two vortex solution by a dashed line.

The mathematical description of this region will be made in more details in the course of E.Sandier [Ser1, Ser2, SS1, SS2, SS3]. 
The onset of superconductivity in decreasing fields (instability of normal solutions and computation of the fields of nucleation) has been analyzed by Bernoff and Sternberg [BS] and del Pino, Felmer, Sternberg [DFS]. Other works concerning the linearized problem include [HM, LP1, LP2] and also [LP3, HP] in dimension 3. Their works provide, as $d$ and $\kappa$ tends to $\infty$, an asymptotic development of $h_{c 3}$, the field at which the normal solution bifurcates to a vortex solution. This is what is called surface superconductivity. In the high kappa limit, their computation yields

$$
h_{c_{3}} \sim \frac{\kappa^{2}}{\lambda_{1}}+C \kappa \kappa_{\max }+o(\kappa)
$$

where $\kappa_{\max }$ is the maximal curvature of the domain and $\lambda_{1}$ is the first eigenvalue of the linearized problem and is approximately equal to 0.59 . In the high $d$ limit, it yields

$$
h_{c_{3}} \sim \frac{\kappa^{2}}{\lambda_{1}}+\frac{C \kappa \kappa_{\max }}{d^{2}}+o\left(\frac{1}{d^{2}}\right)
$$

This expansion is consistent with the work of Saint James and de Gennes [SJdG] who got the first term of this expansion in the case of an infinite plane in one dimension. In two dimensions, one has to take into account the curvature of the cross section. In the case of the disc, the equivalent of expansions (2.2) and (2.3) have been carried out by [BPT] in the limit $\kappa d$ large.

Pan $[\mathrm{P}]$ has rigorously analyzed the state of the material when the magnetic field is further decreased from the nucleation. He proves that the wave function $\psi$ is non zero in a uniform neighborhood of the boundary. It is very important to notice that because of a boundary layer of size $1 / \kappa$, the problem reduces to a $1 \mathrm{~d}$ problem.

Curve $\kappa_{2}(d)$ :

For fixed $\kappa$ above 0.7, when $d$ is increased from 0 , the point $(d, \kappa)$ is first in Region 1 . Then it reaches the critical value $d_{2}(\kappa)$. For $d=d_{2}(\kappa)$, the bifurcation diagram $\|\psi\|_{\infty}$ vs $h$ is decreasing and the superconducting solution bifurcates from the normal solution at $h=h_{*}$. For $d$ a little bigger than $d_{2}(\kappa)$, there is a vortex solution bifurcating from the normal solution close to $h_{*}$. Hence for $d=d_{2}(\kappa)$, at $h=h_{*}$, the linearized problem near the normal state has two eigenfunctions: one without vortices and one with a vortex. This is how uniqueness of solution is lost when increasing $d$, though the assertion needs to be proved mathematically.

Let $D$ be the fixed domain such that $\Omega=d D$. Then a bifurcated solution near the normal state $\left(0, h_{0} a_{0}\right)$ (where $a_{0}$ is such that curl $a_{0}=1$ in $D$ and $a_{0} \cdot n=0$ on $\partial \Omega$ ) is of the form $\left(\varepsilon \phi, h_{0} a_{0}+\varepsilon B\right)$. Let $\omega=h_{0} d$. The second variation of $\mathcal{G}$ near the normal state is

$$
\frac{\partial \mathcal{G}^{2}}{\partial \varepsilon^{2}}\left(\varepsilon \phi, h_{0} a_{0}+\varepsilon B\right)=\frac{1}{d^{2}} \int_{D}\left|\left(i \nabla+\omega^{2} a_{0}\right) \phi\right|^{2}-\kappa^{2} d^{2}|\phi|^{2}+|\operatorname{curl} B|^{2} .
$$

Let

$$
\lambda(\omega)=\inf \left(\int_{D}\left|\left(i \nabla+\omega^{2} a_{0}\right) \phi\right|^{2},\|\phi\|_{L^{2}}=1, \phi \in H^{1}(D, \mathbb{C})\right)
$$


Hence if $\lambda(\omega)>\kappa^{2} d^{2}$, the normal solution is stable, if $\lambda(\omega)<\kappa^{2} d^{2}$, the normal solution is unstable and if $\lambda(\omega)=\kappa^{2} d^{2}$, degenerate stability occurs. For the eigenvalue $\lambda(\omega)=\kappa^{2} d^{2}$, bifurcation of non normal solutions occurs. Thus, one has to study

$$
\left\{\begin{array}{l}
\left(\nabla-i \omega^{2} a_{0}\right)^{2} \phi=\lambda(\omega) \phi \text { in } D \\
\frac{\partial \phi}{\partial n}=0 \text { on } \partial D
\end{array}\right.
$$

with $\lambda(\omega)=\kappa^{2} d^{2}$. For most values of $\kappa$ and $d$, the field $h$ such that the first eigenvalue $\lambda(\omega)$ is equal to $\kappa^{2} d^{2}$ yields a single eigenfunction. In region 1 , the eigenfunction has no vortex while in region 2 , the eigenfunction has a vortex. Thus, the curve $\kappa_{2}(d)$ corresponds to those values of $\kappa$ and $d$ for which the eigenvalue has two different eigenfunctions, one without vortices and one with a vortex. That is, on $\kappa_{2}(d)$, the vortex state starts to exist.

This situation has been studied in the case of a ball in [BPT]. In this case, the solutions of (2.4) with $n$ vortices are of the form $\xi_{n}(r) \exp (i n \theta)$ and have eigenvalue $\lambda(\omega, n)$. In particular in [BPT] they draw the function $\lambda(\omega, n)$ versus $\omega$. The curves $\lambda(\omega, 0)$ and $\lambda(\omega, 1)$ intersect exactly once for $\omega=\omega_{0}$ and $\lambda=\lambda_{0}$. Because of the bifurcation condition $\lambda(\omega)=\kappa^{2} d^{2}$, it implies that $\kappa^{2} d^{2}=\lambda_{0}$, hence the curve $\kappa_{2}(d)$ is of the form $\kappa d=$ constant. It would be interesting to give a rigorous proof that the curves $\lambda(\omega, 0)$ and $\lambda(\omega, 1)$ intersect only once for the case of the disc and for the case of a more general domain. In region 1 , that is below $\kappa_{2}(d)$, the first eigenfunction is simple and leads to a solution without vortex. In region 2 , that is above $\kappa_{2}(d)$, the first eigenfunction is simple and leads to a solution with one vortex, but we expect that there is also an eigenfunction with no vortex for a lower field $h$.

Similarly, the curves $\lambda(\omega, n)$ and $\lambda(\omega, n+1)$ also intersect only once on the numerics of [BPT] which means in our setting that there are curves $\kappa d=C_{n}$ at which the eigenvalue has two eigenfunctions with $n$ and $n+1$ vortices. Above $\kappa d=C_{n}$, a solution with $n+1$ vortices starts to bifurcate from the normal state and below it, a solution with $n$ vortices starts to bifurcate from the normal state, so that the curve $\kappa d=C_{n}$ are the critical curves for the existence of $n+1$ vortices.

In the general case that we are studying, it is totally open to prove that there is a unique value of $\omega$ such that $\lambda(\omega)$ has two eigenvalues, one with a vortex and one without. This would yield to $\kappa_{2}(d)=C / d$, which is what we have found numerically. Moreover, we observe that the field of bifurcation $h_{*}$ satisfies $h_{*} d=\omega$ hence is constant along $\kappa_{2}(d)$.

Taking this analysis of bifurcation a little further allows us to define

$$
\mathcal{H}(\kappa, d)=\left\{h, \text { s.t. } \lambda(\sqrt{h d})=\kappa^{2} d^{2}\right\}
$$

In region 1 , we expect that $\mathcal{H}$ has a single element while in Region 2 , we expect this set to have several elements corresponding to the various branches of solutions with several vortices bifurcating from the normal state. But this analysis is open even in the case of the disc.

Region 3: $\kappa_{4}(d)<\kappa<\kappa_{3}(d)$. 
For parameters in this region, that is large domains and intermediate $\kappa$ (in a relative sense), a typical phase diagram is illustrated in Figure 2.8 with the energy in Figure 2.9. Three solution branches are shown which represent the normal solution, the superconducting solution (solid line) and a solution with a single vortex (dashed line). A profile for one of the vortex solutions is given in Figure 2.1.

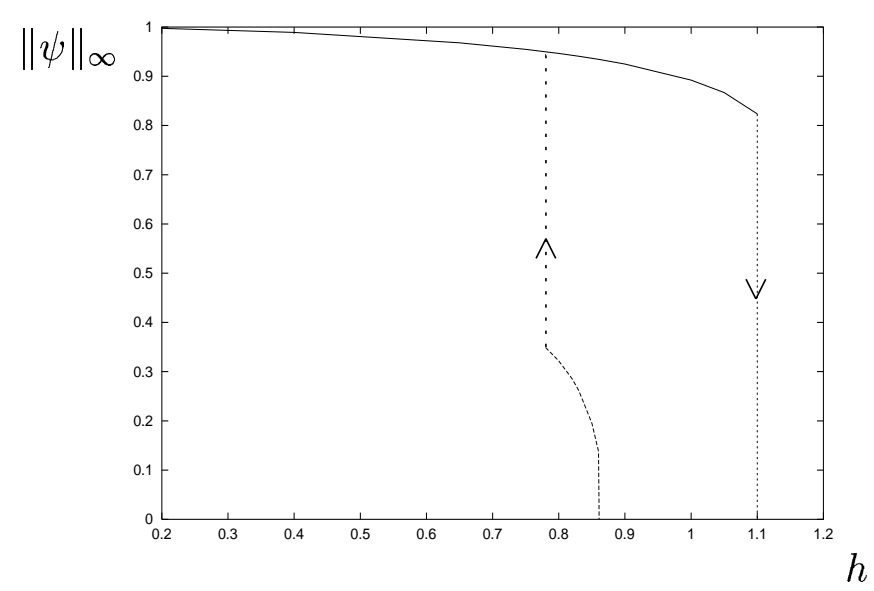

Figure 2.8: The bifurcation curve for $d=6.274 \kappa=0.35$

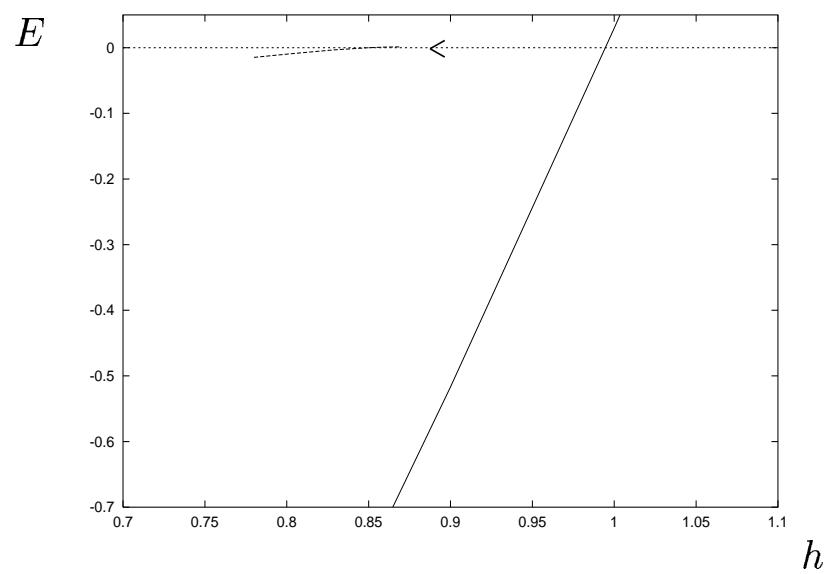

Figure 2.9: The energy for $d=6.274 \kappa=0.35$

The superconducting solution displays a hysteresis behavior as before, but when increasing the field, it turns normal instead of going on the vortex branch as in Region 2 . More precisely, as the field is increased, the superconducting solution loses its global stability, then its local stability and its drops to the normal branch when the super-heating field is reached. Conversely when decreasing the field from the normal state, the solution gets on the vortex branch, though it is only a local minimizer. So the transition when going down the field is of second order, but when going up, there is a hysteresis. When decreasing the field further, the solution jumps to the superconducting branch. The vortex 
solution is never a global minimizer. In fact, our numerical experiments indicate that there are only locally stable vortex solutions.

We note that, for values of the Ginzburg-Landau parameter $\kappa$ in this region, the vortex state has not been frequently studied in the literature, except for superconducting sample with extreme geometrical conditions such as thin films, disks and rings. In the latter cases, the material displays typical type-II behavior for all ranges of $\kappa$ as the Ginzburg-Landau models can be simplified to allow an almost uniform penetration of the magnetic field [CDG]. However, the current study is done for three dimensional infinite cylinders and the simplified models are not directly applicable. In fact, from the plot of the magnetic field given in Figure 2.1, we see that there is considerable variation in the field strength over the cross section.

Curve $\kappa_{3}(d)$ :

Let us call $H_{c}$ the thermodynamic critical field introduced by Ginzburg [G]: the energy of the superconducting solution is equal to the energy of the normal solution at this field (in our nondimensionalization, it means that the energy of the superconducting solution is zero). The curve $\kappa_{3}(d)$ corresponds to the situation where there is a small amplitude vortex solution bifurcating from the normal solution exactly at $H_{c}$. One could hope to determine this curve mathematically.

We notice that as $d$ tends to infinity, $\kappa_{3}(d)$ tends to a finite limit close to 0.4 . A rigorous mathematical justification of this asymptotic behavior remains to be provided. One may notive that this is the same limiting value as in the $1 \mathrm{~d}$ case. One could hope to prove that the bifurcation is determined by the same eigenvalue problem. See [HM].

Region 4: $\kappa<\kappa_{4}(d)$ and $d>d_{1}(\kappa)$.

For parameters in this region, that is $\kappa$ small but domains large enough, the typical bifurcation diagram is illustrated in Figure 2.10 with the energy in Figure 2.11.

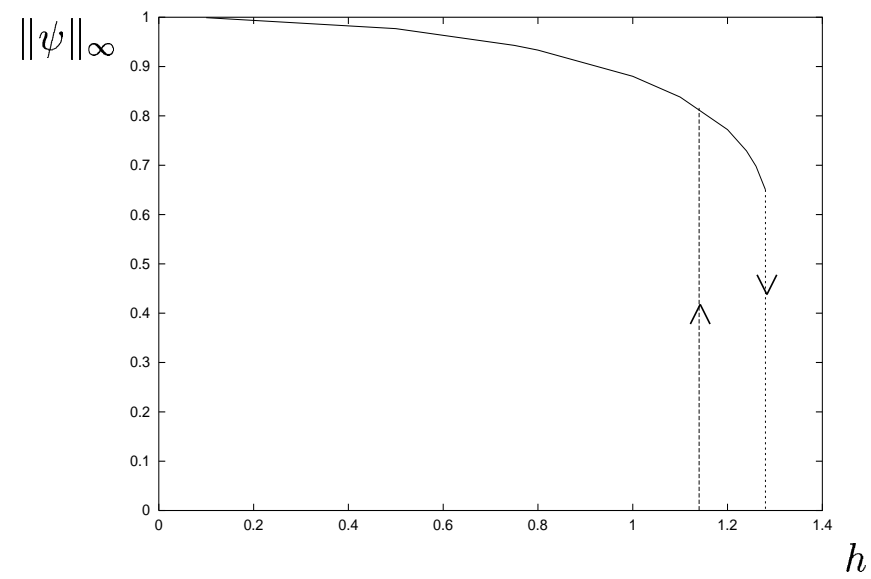

Figure 2.10: The bifurcation curve for $d=4 \kappa=0.3$ 


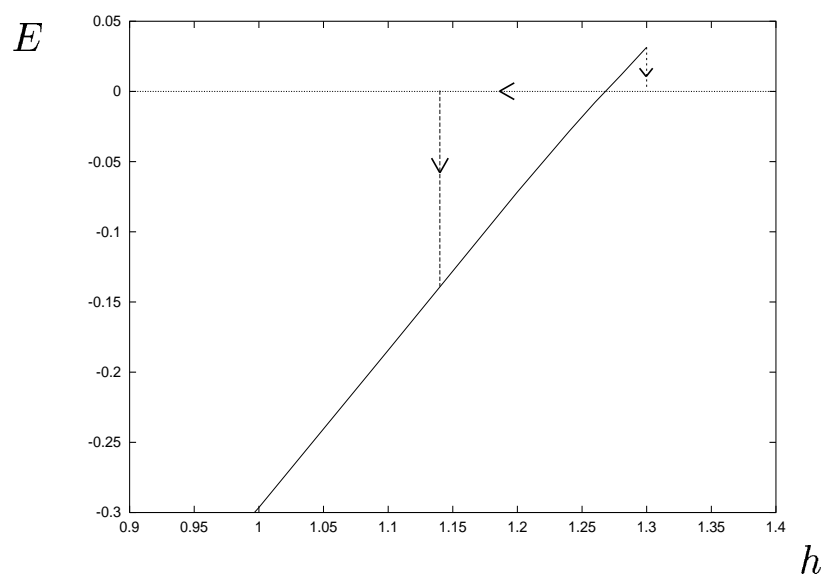

Figure 2.11: The energy for $d=4 \kappa=0.3$

There are superconducting solutions displaying a hysteresis phenomenon and no locally stable vortices. The superconducting solution is not always the global minimizer, but when increasing the field, the sample remains superconducting until reaching a super-heating field $h^{*}$, where the solution becomes normal with a discontinuous transition. Similarly, when decreasing the field, the sample stays normal until the field $h_{*}$ which is less than $h^{*}$, where it turns superconducting by a discontinuous transition. Mathematically, we believe that there is a range of fields, between $h_{*}$ and $h^{*}$ where there are multiple superconducting solutions. This is in analogy with what happens for the one dimensional case. For more rigorous analysis of the one dimensional models, we refer to [AT1].

Next, we also note that there is no locally stable vortex solution in the region. It is wellknown that asymptotically for small $\kappa$, the vortex solution is not energetically favorable, and the material belongs to the typical type-I regime $[\mathrm{G}, \mathrm{T}]$ where the phase transition is characterized by the superconducting/normal interface rather than the vortex state. When varying the field, the superconducting or normal solutions will not turn into a one vortex solution. However, if we do continuation from a vortex solution for a bigger value of $\kappa$ (in Region 3), and decrease $\kappa$, we can still find existence of solutions with vortices when we reduce $\kappa$ to values in Region 4 despite of the instability of vortex solutions. For even smaller $\kappa$, continuations in $\kappa$ or in other parameters from the vortex solutions fails to produce any new vortex solutions. We believe that when decreasing $\kappa$, the vortex solution first loses its stability near the normal solution (on the curve $\kappa_{4}(d)$, but it remains locally stable for $\|\psi\|_{\infty}$ a little higher in the branch. For very small $\kappa$, (especially less than $C / d$ ), we believe that there is no vortex solution at all. This has been proved in [BPT] in the case of a disc. It is an open problem to prove that for fixed $d$ and for $\kappa$ small enough, vortex solutions do not exist.

Curve $\kappa_{1}(d)$ :

If $\kappa$ is fixed below 0.7 and $d$ is increased, then the curve $d_{1}(\kappa)$ is crossed. It remains establish the mathematical existence of this curve. In the particular case where $\kappa$ is very 
small, the order parameter $\psi$ is almost constant, there are no vortices in the domain so that system (2.1) simplifies to

$$
\Delta A=|\psi|^{2} A, \text { in } \Omega \quad \operatorname{curl} A=1 \text { on } \partial \Omega
$$

where $|\psi|$ is a constant that depends on $h_{0}$. The boundary condition for $\psi$ yields $\int|\psi|\left(|\psi|^{2}+\right.$ $\left.h_{0}^{2} A^{2}-1\right)=0$, which is the equation of the bifurcation curve. One has to find the critical $d$ for which the curve $|\psi|\left(h_{0}\right)$ changes direction of bifurcation near $|\psi|=0$, so that the bifurcation goes from stable for small $d$ to unstable for larger $d$. Another way to study this curve is to make the bifurcation analysis near the normal state, described in the analysis of $\kappa_{2}(d)$, which yields to (2.4). Then one would need to take this development to higher order to get the sign of energy of the bifurcated branch. This sign changes on $\kappa_{1}(d)$. The fact that the bifurcation from the normal state is unstable for large $d$ has not been studied.

Curve $\kappa_{4}(d)$ :

Another open problem is to determine the behavior of $\kappa_{4}(d)$ as $d$ tends to infinity. We expect it to be of the order $C / d$ for some constant $C$. We believe that the analysis that we have explained for the curve $\kappa_{2}(d)$ is the same here, that is on $\kappa_{4}(d)$ as well the eigenvalue has two eigenfunctions. The same analysis of the linearized problem needs to be performed. The difference with $\kappa_{2}(d)$ is that the eigenfunction with no vortex is stable on $\kappa_{2}(d)$ and unstable on $\kappa_{4}(d)$.

\section{The point of intersection}

Note that all curves $\kappa_{i}(d)$ intersect at the same point. Indeed the point of intersection of $\kappa_{2}(d)$ and $\kappa_{4}(d)$ has an eigenvalue with two eigenfunctions, one of which (the one without vortices) changes stability. Hence this point also belongs to $\kappa_{1}(d)$ since on $\kappa_{1}(d)$ the stability of the solution without vortex changes. Finally this point belongs to $\kappa_{3}(d)$ since the energy of the bifurcated solution is zero for both eigenfunctions, in particular for the vortex solution. We want to point out that a similar analysis for the intersection of these curves has been performed in [AC2] in the one dimensional setting and it yields to a solvability condition of fourth order at the point of intersection.

\subsection{Uniqueness for small $d$}

Let us now prove rigorous results for region $R_{1}$.

Theorem 2.1 Assume that $D$ is a fixed simply connected bounded domain and let $\Omega=d D$. For any $d_{0}>0$, there exists $d_{1}>0$, such that if $d<\min \left(d_{0}, d_{1} / \kappa\right)$, for any $H_{0}=H_{0}(d, \kappa)$, then any solution of $(G L)$ which is not a normal solution is such that $\psi$ has no zero. Moreover, $\left.|\psi /| \psi\right|_{\infty}-1 \mid \leq C \kappa d$.

We let $H_{0}$ be a function of $d$ and $\kappa$ because it is expected that a superconducting solution exists up to fields $H_{0}$ of the order of $C / d$. Additionally, we prove a symmetry and uniqueness result when the domain is a small disc: 
Theorem 2.2 Let $\Omega$ be a disc of radius $d$. There exist constants $d_{0}$ and $d_{1}$, such that if $d<\min \left(d_{0}, d_{1} / \kappa\right)$, for any $H_{0}=H_{0}(d, \kappa)$, then any solution of $(G L)$ is radially symmetric, that is $\psi(x, y)=\psi(r)$ and $\mathbf{A}(x, y)=A(r) \mathbf{e}_{\theta}$, where $r=\sqrt{x^{2}+y^{2}}$ and this solution is unique up to multiplication of $\psi$ by a constant of modulus 1.

Corollary 2.1 Under the hypotheses of Theorem 2.2, there exists $H_{*}=H_{*}(d, \kappa)$, with $\lim _{d \rightarrow 0} d H_{*}(d, \kappa)=2 \sqrt{2}$ and for $H_{0}<H_{*}$, there exists a unique non normal solution of $(G L)$, while for $H_{0} \geq H_{*}$, the only solutions are the normal solutions.

We make a change of variables $x^{\prime}=x / d, y^{\prime}=y / d$ so that the new variables lie in a domain of unit size $D$. We also define $\mathbf{B}=\kappa d \mathbf{A}$ and $h_{0}=\kappa d^{2} H_{0}$. In the following, we will assume additionnally that

$$
\operatorname{div} \mathbf{B}=0 \quad \text { in } \quad \Omega \quad \text { and } \quad \mathbf{B} \cdot \mathbf{n}=0 \quad \text { on } \quad \partial \Omega .
$$

The equations then become

$$
\left\{\begin{array}{l}
(\nabla-i \mathbf{B})^{2} \psi=\kappa^{2} d^{2} \psi\left(|\psi|^{2}-1\right) \text { in } D \\
\Delta \mathbf{B}=d^{2}\left(\frac{i}{2}\left(\psi^{*} \nabla \psi-\psi \nabla \psi^{*}\right)+\mathbf{B}|\psi|^{2}\right) \text { in } D \\
\frac{\partial \psi}{\partial n}=0 \text { on } \partial D \\
\operatorname{curl} \mathbf{B} \times \mathbf{n}=\mathbf{h}_{0} \times \mathbf{n} \text { on } \partial D
\end{array}\right.
$$

Note that another way of writing the equation for $\mathbf{B}$ is

$$
\Delta \mathbf{B}=-d^{2}(i \psi, \nabla \psi-i \mathbf{B} \psi)
$$

where $(.,$.$) is the real part of the scalar product in \mathbb{C}$.

We allow $h_{0}$ to vary with $d$ and $\kappa$ but we will prove that if there exists a solution, then in fact $h_{0}$ is bounded, that is $H_{0}$ is bounded by $C / \kappa d^{2}$.

The proof consists in obtaining a priori estimates for the solutions $(\psi, \mathbf{B})$.

Proposition 2.1 Fix $p>1$. Assume that $(p s i, \mathbf{B})$ is a non normal solution of $\left(G L_{d}\right)$ and (2.5). For all constants $d_{0}$ and $d_{1}$, if $d<\min \left(d_{0}, d_{1} / \kappa\right)$, then $\psi$ and $\mathbf{B}$ are bounded in $W^{2, p}(D)$ by constants independent of $d$ and $\kappa$. Moreover, for fixed $\kappa$,

$$
\lim _{d \rightarrow 0} h_{0}=0 \quad \text { and } \quad \lim _{d \rightarrow 0}\|\mathbf{B}\|_{W^{2, p}(D)}=0 .
$$

We use these a priori estimates and the Poincaré inequality to derive that $\left\|\nabla \psi /|\psi|_{\infty}\right\|_{\infty}$ and $\left.|\psi /| \psi\right|_{\infty}-1 \mid$ are small, thus $\psi$ has no zero.

Then, we define the functions

$$
\begin{gathered}
\tilde{\psi}(x, y)=\psi(-x, y), \quad \tilde{\mathbf{B}}(x, y)=\left(\begin{array}{c}
-B_{1}(-x, y) \\
B_{2}(-x, y)
\end{array}\right), \\
w(x, y)=\frac{1}{|\psi|_{\infty}}(\psi(x, y)-\tilde{\psi}(x, y)), \quad \mathbf{z}(x)=\mathbf{B}(x, y)-\tilde{\mathbf{B}}(x, y),
\end{gathered}
$$


which satisfy elliptic PDE's with small right hand side terms. Then we use that $\psi$ is nearly constant and hence we get that $w$ and $\mathbf{z}$ are identically zero. This proves Theorem 2.2. We obtain the uniqueness result proving that any solution is necessary a local minimizer of the energy. Finally, we show how our proof can be adapted to the one dimensional case to provide a symmetry result in this setting. 


\section{Chapter 3}

\section{Vortices in Bose Einstein condensates}

\subsection{Introduction}

Bose Einstein condensates owe their name to the prediction of Bose and Einstein in 1925 that for a gas of identical particules without interaction at very low temperature, a macroscopic fraction of the gas is in the state of lowest energy, that is condensed. At that time, the idea was only theoretical. In 1937, superfluid helium was discovered and a link was made with Bose Einstein condensation, but there are major differences between helium, which is a liquid, and the theory of Bose and Einstein which applies to a gas. The first experimental achievement of Bose-Einstein condensates in confined alkali-metal gases was made by American teams in 1995 and was awarded the Nobel prize for physics in 2001 . Since then, there has been a huge experimental and theoretical interest in these systems [BuR, CD, DGPS, MCDW, MCBD]. The study of vortices is one of the key issues. In the ENS experiment [MCDW, MCBD], the trap has a cigar shape and is rotated along its long axis at angular velocity $\Omega$. At small $\Omega$, no modification of the condensate is observed. But at some threshold velocity, a dip of density appears, called a vortex. In[MCBD], it has been observed that when the first vortex is nucleated, the contrast is not $100 \%$ which means that the vortex line is not straight but bending. Numerical computations solving the Gross-Pitaevskii equation [GP] have shown that there is a range of velocities for which the vortex line is indeed bending (see Figure 3.1). At higher velocity, there are more vortices. The aim of this part of the course is to justify these observations theoretically using an appropriate mathematical model.

\subsection{Mathematical formulation}

The condensate is determined by the ellipsoid

$$
\mathcal{D}=\left\{\rho_{\mathrm{TF}}>0\right\} \text { where } \rho_{\mathrm{TF}}=\rho_{0}-\left(x^{2}+\alpha^{2} y^{2}+\beta^{2} z^{2}\right)
$$




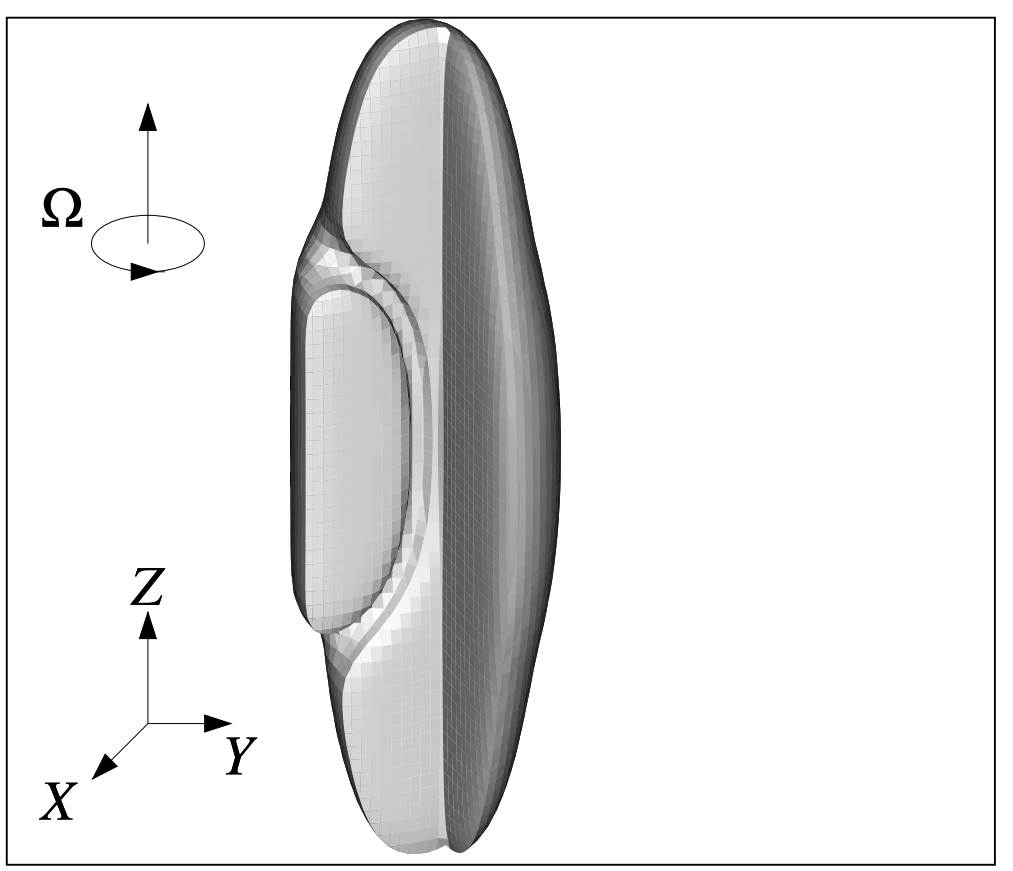

Figure 3.1: Shape of the ground state: vortex bending

Here $\alpha$ and $\beta$ are the geometrical parameters of he trap, $\alpha$ being close to 1 and $\beta$ of order $1 / 15$, which accounts for the elongated shape of $\mathcal{D}$ in Figure 3.1. Moreover

$$
\rho_{0}^{5 / 2}=15 \alpha \beta / 8 \pi
$$

so that

$$
\int_{\mathcal{D}} \rho_{\mathrm{TF}}(\mathbf{r})=1
$$

The trap is rotating at angular velocity $\Omega$ and in the rotating frame, all the particules are assumed to be condensed in the same state described by a wave function, which minimizes the Gross-Pitaevskii energy:

$$
E_{\varepsilon}(u)=\int_{\mathcal{D}} \frac{1}{2}|\nabla u|^{2}+\boldsymbol{\Omega} \cdot(i u, \nabla u \times \mathbf{r})+\frac{1}{4 \varepsilon^{2}}\left(\rho_{\mathrm{TF}}(\mathbf{r})-|u|^{2}\right)^{2} \quad u \in H_{0}^{1}(\mathcal{D}), \quad \int_{\mathcal{D}}|u|^{2}=1 .
$$

Here, for any complex quantities $u$ and $v$ and their complex conjugates $\bar{u}$ and $\bar{v},(u, v)=$ $(u \bar{v}+\bar{u} v) / 2$. The first term is the kinetic energy, then there is the term due to rotation, the term in $|u|^{4}$ accounts for the interactions and $\rho_{\mathrm{TF}}|u|^{2}$ is the trapping term. The parameter $\varepsilon$ is a non dimensional parameter of order $10^{-2}$. The velocity $\Omega$ has to be such that $\Omega<1 / \varepsilon$, so that the trapping potential is stronger than the inertial potential. For a more precise derivation of the physical model, see [ADu2]. We have written the energy in such a way that the analogy with the Ginzburg-Landau becomes clear: the magnetic field is replaced by rotation and the trapping $\rho_{\mathrm{TF}}$ plays the role of a pinning term. 
Note that a critical point $u$ of $E_{\varepsilon}$ is a solution of

$$
-\Delta u+2 i(\boldsymbol{\Omega} \times \mathbf{r}) \cdot \nabla u=\frac{1}{\varepsilon^{2}} u\left(\rho_{\mathrm{TF}}-|u|^{2}\right)+\mu_{\varepsilon} u \text { in } \mathcal{D},
$$

with $u=0$ on $\partial \mathcal{D}$ and $\mu_{\varepsilon}$ is the Lagrange multiplier. The specific choice of $\rho_{0}$ in (3.1) will imply that the term $\mu_{\varepsilon} u$ is negligible in front of $\rho_{\mathrm{TF}} u / \varepsilon^{2}$.

We have set the framework of study of our energy. We want to determine the properties of its minimizers according to the value of $\Omega$. We will make an asymptotic expansion of the energy taking into account that $\varepsilon$ is small to obtain a simpler form of the energy which only depends on the shape of the vortex lines. Then we check numerically and theoretically that our characterization leads to solutions with a bent vortex for a range of values of the rotational velocity which are consistent with the ones obtained numerically.

\subsection{Asymptotic expansion of the energy}

Our aim is to decouple the energy into 3 terms: a part coming from the profile of the solution without vortices, a vortex contribution and a term due to rotation.

In Figure 3.2, 3.3, we have plotted $\left|u_{\varepsilon}\right|$ as a function of $x$ and $y$, at $z=0$, for $\Omega=0$ and 20. The solution has the same profile except close to vortices and $\left|u_{\varepsilon}\right|^{2}$ is close to $\rho_{\mathrm{TF}}$ except near the boundary.

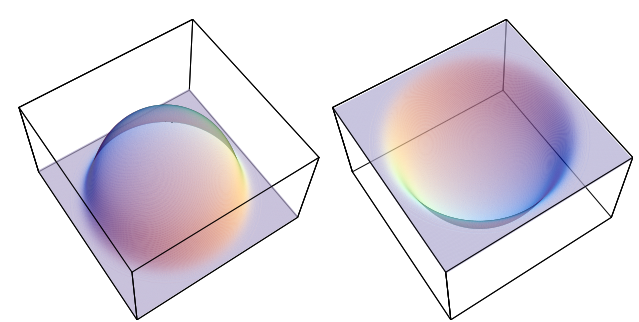

Figure 3.2: $|u|$ for $\Omega=0$.
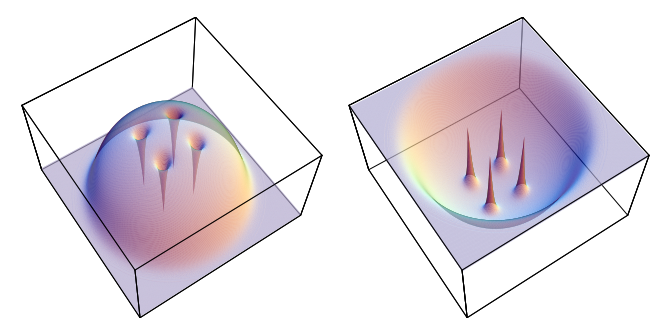

Figure 3.3: $|u|$ for $\Omega=20$. 


\subsubsection{The profile of the solution}

Firstly, we are interested in the profile of solutions so that we will study solutions without vortices. Thus we consider functions of the form $\eta=f e^{i S}, f$ is real and does not vanish in the interior of $\mathcal{D}$. We consider first minimizing $E_{\varepsilon}$ over such functions without imposing the constraint that the norm is 1 , that is, $f$ and $S$ minimize

$$
\mathcal{E}_{\varepsilon}(f, S)=\int_{\mathcal{D}} \frac{1}{2}|\nabla f|^{2}+\frac{1}{4 \varepsilon^{2}}\left(\rho_{\mathrm{TF}}-f^{2}\right)^{2}+\frac{1}{2} \int f^{2}|\nabla S-\boldsymbol{\Omega} \times \mathbf{r}|^{2}-f^{2} \Omega^{2} r^{2},
$$

where $\mathbf{r}=x \mathbf{e}_{x}+y \mathbf{e}_{y}$. We have $f_{\varepsilon}=0$ on $\partial \mathcal{D}$ and

$$
\begin{gathered}
-\Delta f_{\varepsilon}+f_{\varepsilon} \nabla S_{\varepsilon}\left(\nabla S_{\varepsilon}-2 \boldsymbol{\Omega} \times \mathbf{r}\right)=\frac{1}{\varepsilon^{2}} f_{\varepsilon}\left(\rho_{\mathrm{TF}}-f_{\varepsilon}^{2}\right) \text { in } \mathcal{D}, \\
\operatorname{div}\left(f_{\varepsilon}^{2}\left(\nabla S_{\varepsilon}-\boldsymbol{\Omega} \times \mathbf{r}\right)\right)=0 .
\end{gathered}
$$

From the first equation, one can derive that $f_{\varepsilon}^{2}$ tends to $\rho_{\mathrm{TF}}$ on every compact subset of $\mathcal{D}$. Indeed there is a boundary layer since $\sqrt{\rho_{\mathrm{TF}}}$ is not in $H^{1}(\mathcal{D})$. the boundary layer is of size $\varepsilon^{2 / 3}$ and after proper nondimensionalization, is given by a solution of a Painleve type equation:

$$
v^{\prime \prime}=v\left(v^{2}-2 \sqrt{\rho_{0}} x\right) \quad v(-\infty)=0 \quad v(x) \approx \sqrt{2 \sqrt{\rho_{0}} x} \text { as } x \rightarrow \infty .
$$

The continuity equation (3.7) implies that there exists $\Xi_{\varepsilon}$ in $H^{2} \cap H_{0}^{1}$ such that

$$
f_{\varepsilon}^{2}\left(\nabla S_{\varepsilon}-\boldsymbol{\Omega} \times \mathbf{r}\right)=\Omega \operatorname{curl} \boldsymbol{\Xi}_{\varepsilon}
$$

One can think of $\boldsymbol{\Xi}_{\varepsilon}$ as the equivalent of a stream function in the case of fluid vortices. So $\boldsymbol{\Xi}_{\varepsilon}$ is the unique solution of

$$
\operatorname{curl}\left(\frac{1}{f_{\varepsilon}^{2}} \operatorname{curl} \boldsymbol{\Xi}_{\varepsilon}\right)=-2 \text { in } \mathcal{D}, \quad \boldsymbol{\Xi}_{\varepsilon}=0 \text { on } \partial \mathcal{D} .
$$

In the special case where the cross section of $\mathcal{D}$ is a disc (that is $\alpha=1$ ), the minimum of (3.5) is reached for $\nabla S=0$ but this is not the case if the cross section is an ellipse and there is a non trivial solution of (3.7). When $\varepsilon$ is small, $f_{\varepsilon}^{2}$ tends to $\rho_{\mathrm{TF}}$ on every compact subset of $\mathcal{D}$ and the function $\boldsymbol{\Xi}_{\varepsilon}$ given by (3.8) or (3.9) is approximated by the unique solution $\Xi$ of

$$
\operatorname{curl}\left(\frac{1}{\rho_{\mathrm{TF}}} \operatorname{curl} \Xi\right)=-2 \text { in } \mathcal{D}, \quad \Xi=0 \text { on } \partial \mathcal{D} .
$$

One can easily get that

$$
\Xi(x, y)=-\rho_{\mathrm{TF}}^{2}(x, y) /\left(2+2 \alpha^{2}\right) \mathbf{e}_{z} .
$$

Using (3.8), we can define $S_{0}$, the limit of $S_{\varepsilon}$, to be the solution of $\rho_{\mathrm{TF}}\left(\nabla S_{0}-\boldsymbol{\Omega} \times \mathbf{r}\right)=$ $\Omega$ curl $\Xi$ with zero value at the origin. We have $S_{0}=C \Omega x y$ with $C=\left(\alpha^{2}-1\right) /\left(\alpha^{2}+1\right)$. We see that $S_{0}$ vanishes when $\alpha=1$, that is when the cross-section is a disc. Otherwise, the solution $\eta_{\varepsilon}$ has a phase which is globally defined. 


\subsubsection{Decoupling the energy}

One now needs to add the vortex contribution on the profile of solutions. Let $\eta_{\varepsilon}=f_{\varepsilon} e^{i S_{\varepsilon}}$ be the vortex free minimizer of $E_{\varepsilon}$ discussed previously without imposing the constraint on the norm of $u_{\varepsilon}$. Let $u_{\varepsilon}$ be a configuration that will minimize $E_{\varepsilon}$ and let $v_{\varepsilon}=u_{\varepsilon} / \eta_{\varepsilon}$. Since $\eta_{\varepsilon}$ satisfies the Gross Pitaevskii equation (3.6)-(3.7), we have

$$
\int_{\mathcal{D}}\left(\left|v_{\varepsilon}\right|^{2}-1\right)\left(-\frac{1}{2} \Delta f_{\varepsilon}^{2}-\frac{1}{\varepsilon^{2}} f_{\varepsilon}^{2}\left(\rho_{\mathrm{TF}}-f_{\varepsilon}^{2}\right)+\left|\nabla f_{\varepsilon} e^{i S_{\varepsilon}}\right|^{2} \quad-2 f_{\varepsilon}^{2}\left(\nabla S_{\varepsilon} \cdot \boldsymbol{\Omega} \times \mathbf{r}\right)\right)=0 .
$$

This trick was introduced in $[\mathrm{LM}]$ and leads to the following decoupling of the energy $E_{\varepsilon}\left(u_{\varepsilon}\right)$ :

$$
E_{\varepsilon}\left(u_{\varepsilon}\right)=E_{\varepsilon}\left(\eta_{\varepsilon}\right)+G_{\eta_{\varepsilon}}\left(v_{\varepsilon}\right)+I_{\eta_{\varepsilon}}\left(v_{\varepsilon}\right)
$$

where

$$
G_{\eta_{\varepsilon}}\left(v_{\varepsilon}\right)=\int_{\mathcal{D}} \frac{1}{2}\left|\eta_{\varepsilon}\right|^{2}\left|\nabla v_{\varepsilon}\right|^{2}+\frac{\left|\eta_{\varepsilon}\right|^{4}}{4 \varepsilon^{2}}\left(1-\left|v_{\varepsilon}\right|^{2}\right)^{2}
$$

is the energy of vortices and

$$
I_{\eta_{\varepsilon}}\left(v_{\varepsilon}\right)=\int_{\mathcal{D}}\left|\eta_{\varepsilon}\right|^{2}\left(\nabla S_{\varepsilon}-\boldsymbol{\Omega} \times \mathbf{r}\right) \cdot\left(i v_{\varepsilon}, \nabla v_{\varepsilon}\right)
$$

is the angular momentum of vortices. The first term in $E_{\varepsilon}\left(\eta_{\varepsilon}\right)$ is independent of the solution $u_{\varepsilon}$, so we have to compute the next two and find for which configuration $u_{\varepsilon}$ the minimum is achieved. We use that at zero order $\left|\eta_{\varepsilon}\right|^{2}$ is approximated by $\rho_{\mathrm{TF}}$ when $\varepsilon$ is small so that we can estimate $G_{\eta_{\varepsilon}}$ by $G_{\sqrt{\rho_{\mathrm{TF}}}}=G_{\varepsilon}$ and $I_{\eta_{\varepsilon}}$ by $I_{\sqrt{\rho}_{\mathrm{TF}}}=I_{\varepsilon}$. The mathematical techniques to approximate $G_{\varepsilon}$ have been introduced in [BBH] in dimension 2 and in [Ri] in dimension 3. The vortices will be tubes of size $\varepsilon$ around which $v_{\varepsilon}$ has a degree. Away from the tubes, $\left|v_{\varepsilon}\right|$ is very close to 1 and only the phase of $v_{\varepsilon}$ is of influence. In the vortex core, the profile of $v_{\varepsilon}$ is given by the cubic NLS equation. Our aim is to estimate the energy of $u_{\varepsilon}$ depending on the vortex tubes.

\section{$3.42 \mathrm{D}$ case}

We first consider a situation in which the confinement along the $z$ axis is so strong that the motion of particles along this axis is frozen in the ground state of the strong harmonic potential: hence the wave function is factorized into a component purely dependent on $z$ and another one which depends on the radial coordinates $(x, y)$ and it minimizes $E_{\varepsilon}$, but with $\rho_{\mathrm{TF}}=\rho_{0}-x^{2}-\alpha^{2} y^{2}$ and $\rho_{0}^{2}=2 \alpha / \pi$.

The energy of vortices $G_{\varepsilon}$ is estimated as in $[\mathrm{BBH}]$, taking into account the weight $\left|\eta_{\varepsilon}\right|^{2}$ to obtain a leading order term in $|\log \varepsilon|$ and an interaction term. The integral $I_{\varepsilon}$ is computed via an integration by part. One can prove that as $\varepsilon$ goes to 0 , the vortices tend to the origin and they are all singly quantized vortices, that is $d_{i}=1$. The proof goes as in [Ser1]: to show it, one may define a test function that consists in putting the $n$ vortices 
on a polygon centered at the origin of size $1 / \sqrt{\Omega}$ in $x$ and $1 / \alpha \sqrt{\Omega}$ in $y$, and check that this has a lower energy than any configuration with multiple vortices. Using a special ansatz, it has been proved in [CD] that the multiply quantized vortices are unstable. In fact, it is a physical signature of the harmonic trapping potentials that the minimizers have only singly quantized vortices. When other trapping potentials are used, it is possible to have minimizers with multiply quantized vortices $\left(d_{i}>1\right)$.

We let $\tilde{p}_{i}$ be the location of vortices with coordinates $\left(\tilde{x}_{i}, \tilde{y}_{i}\right)$ be such that $\tilde{x}_{i}=x_{i} \sqrt{\Omega}$ and $\tilde{y}_{i}=\alpha y_{i} \sqrt{\Omega}$. This allows us to estimate the energy of a solution with $n$ vortices centered at $\tilde{p}_{i}$ :

$$
E_{\varepsilon}(u)-E_{\varepsilon}\left(\eta_{\varepsilon}\right)=\pi n \rho_{0}\left(|\log \varepsilon|-\frac{\Omega \alpha}{1+\alpha^{2}}\right)+\frac{\pi}{2}\left(n^{2}-n\right) \rho_{0} \log \Omega+w\left(\tilde{p}_{1}, \ldots, \tilde{p}_{n}\right)+C_{n}+o(1)
$$

where $C_{n}$ is a constant that depends on $n$ and $\alpha$ and

$$
\begin{aligned}
& w\left(\tilde{p}_{1}, \ldots, \tilde{p}_{n}\right)=-\pi \rho_{0} \sum_{i \neq j} \log \left(\left|\tilde{x}_{i}-\tilde{x}_{j}\right|^{2}+\frac{\left|\tilde{y}_{i}-\tilde{y}_{j}\right|^{2}}{2 \alpha^{2}}\right) \\
& +\pi \rho_{0} \sum_{i}\left(\tilde{x}_{i}^{2}+\tilde{y}_{i}^{2}\right)\left(\frac{2}{1+\alpha^{2}}-\frac{|\log \varepsilon|}{2 \Omega \rho_{0}}\right) .
\end{aligned}
$$

Physically speaking, the first term in (3.15) gives the repulsive interaction between the vortices and the second term illustrate the competition of the restoring and centrifugal forces [MCBD, MCDW]. The location of vortices is determined by the minimum of $w$. Recall that $\rho_{0}^{2}=2 \alpha / \pi$. For fixed $\alpha, w$ is of order 1 , hence is of lower order than the previous terms. Then the critical angular velocity for the existence of $n$ vortices can be computed from (3.14)

$$
\Omega_{n}=\left(1+\alpha^{2}\right) \sqrt{\frac{\pi}{2 \alpha}}\left(|\log \varepsilon|+\frac{(n-1)}{2} \log \left(\left(1+\alpha^{2}\right) \sqrt{\frac{\pi}{2 \alpha}}|\log \varepsilon|\right)\right),
$$

The location of vortices is determined by the minimum of $w$ or equivalently by the minimum of

$$
-\sum_{i \neq j} \log \left(\left|\tilde{x}_{i}-\tilde{x}_{j}\right|^{2}+\frac{\left|\tilde{y}_{i}-\tilde{y}_{j}\right|^{2}}{\alpha^{2}}\right)
$$

under the constraint $\sum_{i} \tilde{x}_{i}^{2}+\tilde{y}_{i}^{2}=$ cst. For $\alpha=1$, this has been studied by [GS]. When $\alpha \neq 1$, it is an open question. When $n=2$ or 3 (and $\alpha$ not close to 1 ), the vortices are on the longest axis of the ellipse.

\subsection{D case}

In the general case, we want to estimate $G_{\varepsilon}\left(v_{\varepsilon}\right)$ and $I_{\varepsilon}\left(v_{\varepsilon}\right)$ to get an approximate expression for (3.13). We will do it in the case $u_{\varepsilon}$ has a single vortex tube tending to the curve $\gamma$. 


\subsubsection{Estimate of $G_{\varepsilon}\left(v_{\varepsilon}\right)$}

We want to estimate

$$
G_{\varepsilon}\left(v_{\varepsilon}\right)=\int_{\mathcal{D}} \frac{1}{2}\left|\rho_{\mathrm{TF}}\right|\left|\nabla v_{\varepsilon}\right|^{2}+\frac{\left|\rho_{\mathrm{TF}}\right|^{2}}{4 \varepsilon^{2}}\left(1-\left|v_{\varepsilon}\right|^{2}\right)^{2}
$$

We define

$$
T_{\lambda \varepsilon}=\{x \in \mathcal{D} \text { s.t. } \operatorname{dist}(x, \gamma) \leq \lambda \varepsilon\}
$$

and assume that $\lambda \varepsilon$ is small, $\lambda$ being our matching parameter to be fixed later on. Then we split $G_{\varepsilon}$ into two integrals: one in $T_{\lambda \varepsilon}$, the energy of the vortex core and the other in $\mathcal{D} \backslash T_{\lambda \varepsilon}$, the energy away from the vortex core.

\section{Estimate near the vortex core}

We are going to estimate $G_{\varepsilon}$ in $T_{\lambda \varepsilon}$. At each point $\gamma(t)$ of $\gamma$, we define $\Pi^{-1}(\gamma(t))$ to be the plane orthogonal to $\gamma$ at $\gamma(t)$. Since $\lambda \varepsilon$ is small, we assume that $\rho_{\mathrm{TF}}$ is constant in $\Pi^{-1}(\gamma(t)) \cap T_{\lambda \varepsilon}$ and we call the value $\rho_{t}=\rho_{\mathrm{TF}}(\gamma(t))$. We want to compute

$$
\int_{T_{\lambda \varepsilon}} \frac{1}{2} \rho_{\mathrm{TF}}\left|\nabla v_{\varepsilon}\right|^{2}+\frac{\rho_{\mathrm{TF}}^{2}}{4 \varepsilon^{2}}\left(1-\left|v_{\varepsilon}\right|^{2}\right)^{2} \simeq \int_{\gamma} \frac{\rho_{t}}{2} \int_{\Pi^{-1}(\gamma(t)) \cap T_{\lambda \varepsilon}}\left|\nabla v_{\varepsilon}\right|^{2}+\frac{\rho_{t}}{2 \varepsilon^{2}}\left(1-\left|v_{\varepsilon}\right|^{2}\right)^{2} .
$$

This computation is valid as long as $k \lambda \varepsilon$ is small, where $k$ is the curvature of $\gamma$. The zero order approximation of $v_{\varepsilon}$ is given by $u_{1}\left(r \sqrt{\rho_{t}} / \varepsilon\right)$, where $u_{1}(r, \theta)=f_{1}(r) e^{i \theta}$ is the solution with a single zero at the origin of the cubic NLS equation

$$
\Delta u+u\left(1-|u|^{2}\right)=0 \text { in } \mathbb{R}^{2} .
$$

Thus,

$$
\begin{aligned}
& \int_{\Pi^{-1}(\gamma(t)) \cap T_{\lambda \varepsilon}}\left|\nabla v_{\varepsilon}\right|^{2}+\frac{\rho_{t}}{2 \varepsilon^{2}}\left(1-\left|v_{\varepsilon}\right|^{2}\right)^{2} \\
\simeq \quad & \int_{B_{\lambda \varepsilon}}\left|\nabla\left(f_{1}\left(r \sqrt{\frac{\rho_{\mathrm{TF}}}{\varepsilon^{2}}}\right) e^{i \theta}\right)\right|^{2}+\frac{\rho_{t}}{2 \varepsilon^{2}}\left(1-f_{1}^{2}\left(r \sqrt{\frac{\rho_{\mathrm{TF}}}{\varepsilon^{2}}}\right)\right)^{2} \\
= & \int_{B_{\lambda \sqrt{\rho t}}}\left|\nabla u_{1}\right|^{2}+\frac{1}{2}\left(1-\left|u_{1}\right|^{2}\right)^{2} \\
\simeq \quad & c_{*}+2 \pi \log \left(\lambda \sqrt{\rho_{t}}\right)
\end{aligned}
$$

where

$$
c_{*}=\int_{\mathbb{R}^{2}} f_{1}^{\prime 2}+\frac{1}{2}\left(1-f_{1}^{2}\right)^{2}+\int_{\mathbb{R}^{2} \backslash B_{1}} \frac{f_{1}^{2}-1}{r^{2}}+\int_{B_{1}} \frac{f_{1}^{2}}{r^{2}} .
$$

The last line of (3.18) would be an equality if the first two integrals in the expression of $c_{*}$ were taken in $B_{\lambda \sqrt{\rho_{t}}}$ instead of $\mathbb{R}^{2}$. This approximation is correct if $\lambda \sqrt{\rho_{t}}$ is large (in fact bigger than 3 is enough).

The final estimate of this section is

$$
G_{\varepsilon}\left(v_{\varepsilon}\right)_{\mid T_{\lambda \varepsilon}} \simeq \int_{\gamma} \rho_{\mathrm{TF}}\left(\frac{c_{*}}{2}+\pi \log \left(\lambda \sqrt{\rho_{\mathrm{TF}}}\right)\right) d l
$$




\section{Estimate away from the vortex core}

We are going to estimate $G_{\varepsilon}$ in $\mathcal{D} \backslash T_{\lambda \varepsilon}$. In this region $\left|v_{\varepsilon}\right| \simeq 1$, and we have seen that $\lambda \sqrt{\rho_{t}}$ is large, so that only the kinetic energy of the phase has a contribution.

$$
\int_{\mathcal{D} \backslash T_{\lambda \varepsilon}} \frac{1}{2} \rho_{\mathrm{TF}}\left|\nabla v_{\varepsilon}\right|^{2}+\frac{\rho_{\mathrm{TF}}^{2}}{4 \varepsilon^{2}}\left(1-\left|v_{\varepsilon}\right|^{2}\right)^{2} \simeq \int_{\mathcal{D} \backslash T_{\lambda \varepsilon}} \frac{1}{2} \rho_{\mathrm{TF}}\left|\nabla \phi_{\varepsilon}\right|^{2},
$$

where $\phi_{\varepsilon}$ is the phase of $v_{\varepsilon}$. Of course, $\phi_{\varepsilon}$ is not defined everywhere. We let $\Psi$ be a stream function that is $\operatorname{div} \Psi=0$ and

$$
\operatorname{curl} \Psi=\rho_{\mathrm{TF}} \nabla \phi
$$

Then $\Psi$ is the unique solution of

$$
\operatorname{curl}\left(\frac{1}{\rho_{\mathrm{TF}}} \operatorname{curl} \Psi\right)=2 \pi \vec{\delta}_{\gamma}, \Psi=0 \text { on } \partial \mathcal{D}
$$

where $\vec{\delta}_{\gamma}$ is the vectorial Dirac measure along $\gamma$, that is for a vectorial test function $\mathbf{w}$,

$$
<\vec{\delta}_{\gamma}, \mathbf{w}>=\int_{\gamma} \mathbf{w} \cdot d l
$$

while $\delta_{\gamma}$ is the Dirac measure along $\gamma$. Thus,

$$
\int_{\mathcal{D} \backslash T_{\lambda \varepsilon}} \frac{1}{2} \rho_{\mathrm{TF}}\left|\nabla \phi_{\varepsilon}\right|^{2}=\int_{\mathcal{D} \backslash T_{\lambda \varepsilon}} \frac{1}{2 \rho_{\mathrm{TF}}}|\operatorname{curl} \Psi|^{2}=-\frac{1}{2} \int_{\partial T_{\lambda \varepsilon}} \boldsymbol{\Psi} \cdot \nabla \phi_{\varepsilon} \times \nu
$$

where $\nu$ is the outward unit normal to the tube $T_{\lambda \varepsilon}$. We will see that $\Psi$ is almost constant at a distance $\lambda \varepsilon$ from $\gamma$ and we call this value $\boldsymbol{\Psi}_{\lambda \varepsilon}(\gamma)$. Since the vortex line has a winding number $2 \pi$,

$$
\int_{\mathcal{D} \backslash T_{\lambda \varepsilon}} \frac{1}{2} \rho_{\mathrm{TF}}\left|\nabla \phi_{\varepsilon}\right|^{2} \simeq \pi \int_{\gamma} \boldsymbol{\Psi}_{\lambda \varepsilon}(\gamma) \cdot d l
$$

We have to compute $\boldsymbol{\Psi}$ on $\partial T_{\lambda \varepsilon}$. The computation is inspired by the paper of Svidzinsky and Fetter [SvF2]. It follows from (3.21) that $\boldsymbol{\Psi}$ satisfies

$$
-\Delta \Psi-\frac{\nabla \rho_{\mathrm{TF}}}{\rho_{\mathrm{TF}}} \times \operatorname{curl} \Psi=2 \pi \rho_{\mathrm{TF}} \vec{\delta}_{\gamma}
$$

Let $x_{0} \in \gamma$. We denote by $\mathbf{e}_{3}=\dot{\gamma}\left(x_{0}\right)$ and $\left(\mathbf{e}_{1}, \mathbf{e}_{2}, \mathbf{e}_{3}\right)$ an orthogonal base in local coordinates. Then $\boldsymbol{\Psi}$ has coordinates $\psi_{i}$ in $\mathbf{e}_{i}$ and the variations of $\psi_{3}$ are the only ones of influence in the equation for $\boldsymbol{\Psi}$, since we want to compute $\boldsymbol{\Psi} \cdot d l$. In the equation for $\boldsymbol{\Psi}$, we neglect the terms in $\nabla \psi_{1}$ and $\nabla \psi_{2}$ in front of $\nabla \psi_{3}$ and we get

$$
-\Delta \psi_{3}+\frac{\nabla \bar{\rho}_{T F}}{\bar{\rho}_{T F}} \cdot \nabla \psi_{3}=2 \pi \rho_{\mathrm{TF}} \delta_{\gamma}
$$


where $\bar{\rho}_{T F}\left(x^{1}, x^{2}\right)=\rho_{\mathrm{TF}}\left(x^{1}, x^{2}, x_{0}^{3}\right)$. Let $\xi=\psi_{3} / \sqrt{\bar{\rho}_{T F}}$. Then it follows from (3.23) that $\xi$ satisfies

$$
-\Delta \xi+\mu \xi=2 \pi \sqrt{\rho_{\mathrm{TF}}} \delta_{\gamma}
$$

where

$$
\mu=\sqrt{\bar{\rho}_{T F}} \Delta \frac{1}{\sqrt{\bar{\rho}_{T F}}}=\sqrt{\rho_{\mathrm{TF}}} \Delta_{\perp} \frac{1}{\sqrt{\rho_{\mathrm{TF}}}} .
$$

Here $\Delta_{\perp}$ is the Laplacian in the plane perpendicular to $\mathbf{e}_{3}=\dot{\gamma}\left(x_{0}\right)$. If the cross-section of the condensate $\mathcal{D}$ is a disc one can compute $\mu$. We denote by $\theta$ the angle of $\mathbf{e}_{3}$ that is $\mathbf{e}_{3}=\cos \theta \mathbf{e}_{r}+\sin \theta \mathbf{e}_{z}$ and $(r, z)$ are the coordinates of $x_{0}$ in the original frame. Then

$$
\mu=\frac{\left(1+\sin ^{2} \theta\right)+\beta^{2} \cos ^{2} \theta}{\rho_{\mathrm{TF}}}+\frac{3\left(r \sin \theta-\beta^{2} z \cos \theta\right)^{2}}{\rho_{\mathrm{TF}}^{2}} .
$$

Note that $\mu>0$. In fact our numerical computations even yield $\mu>7$. Our aim is now to give an approximate expression for $\xi$. We locally approximate the curve $\gamma$ near the point $x_{0}$ by the parabola $x=k z^{2} / 2$, where $k$ is the curvature of $\gamma$ at $x_{0}$. This is where we use the same ideas as in [SvF2]. Note that in our approximations, we are only taking into account the shape of $\gamma$ close to $x_{0}$. The justification for this relies on the fact that $\mu>7$ as our numerics show. Indeed if we solve

$$
-\Delta X+\mu X=f
$$

where $f$ is supported at a distance $d$ of $x_{0}$. Then using the Green function, we find that

$$
|X| \leq \frac{e^{-\sqrt{\mu} d}}{4 \pi \mu^{3} d} .
$$

In particular, for $d=0.1$, this gives an error less than $10^{-3}$. This is to be compared to the Euler constant and our approximation is reasonable. We rewrite (3.24) in local coordinates to get

$$
-\Delta_{\perp} \xi+k \partial_{x_{1}} \xi+\mu \xi=2 \pi \sqrt{\rho_{\mathrm{TF}}\left(x_{0}\right)} \delta_{\mathbf{e}_{3}},
$$

where $\delta_{\mathbf{e}_{3}}$ is the Dirac mass supported along the line $\mathbf{e}_{3}$ and $\mathbf{e}_{1}$ is the normal to the vortex line $\gamma$. Thus

$$
-\Delta\left(e^{\frac{-k x_{1}}{2}} \xi\right)+\left(\left(\frac{k}{2}\right)^{2}+\mu\right)\left(e^{\frac{-k x_{1}}{2}} \xi\right)=2 \pi \sqrt{\rho_{\mathrm{TF}}\left(x_{0}\right)} \delta_{\mathbf{e}_{3}} .
$$

The solution of this equation is

$$
\sqrt{\rho_{\mathrm{TF}}\left(x_{0}\right)} K_{0}\left(\sqrt{\mu+\frac{k^{2}}{4}} \operatorname{dist}(x, \gamma)\right)
$$

where $K_{0}$ is a modified Bessel function. In particular, $K_{0}(x) \simeq-\log \left(e^{C_{0}} x / 2\right)$ for small $x$ where $C_{0} \simeq 0.577$ is the Euler constant. Hence, we deduce

$$
\Psi(x) \simeq-\rho_{\mathrm{TF}} \log \left(\frac{e^{C_{0}}}{2} \sqrt{\mu+\frac{k^{2}}{4}} \operatorname{dist}(x, \gamma)\right) \dot{\gamma} .
$$


Thus we conclude by the estimate for $G_{\varepsilon}\left(v_{\varepsilon}\right)$ in $\mathcal{D} \backslash T_{\lambda \varepsilon}$

$$
G_{\varepsilon}\left(v_{\varepsilon}\right)_{\mid \mathcal{D} \backslash T_{\lambda \varepsilon}} \simeq-\pi \int_{\gamma} \rho_{\mathrm{TF}} \log \left(\frac{e^{C_{0}}}{2} \sqrt{\mu+\frac{k^{2}}{4}} \lambda \varepsilon\right) d l .
$$

Here we have used that $\lambda \varepsilon$ is sufficiently small. In the previous section, we needed $\lambda \sqrt{\rho_{t}}$ large. The existence of $\lambda$ is justified by the fact that $\sqrt{\rho_{\mathrm{TF}}} / \varepsilon$ is much bigger than 1 , except very close to the boundary. But in this region, the contribution of the energy is negligible.

\subsubsection{Estimate of $I_{\varepsilon}\left(v_{\varepsilon}\right)$}

We want to estimate

$$
I_{\varepsilon}\left(v_{\varepsilon}\right)=\int_{\mathcal{D}} \rho_{\mathrm{TF}}\left(\nabla S_{\varepsilon}-\mathbf{\Omega} \times \mathbf{r}\right) \cdot\left(i v_{\varepsilon}, \nabla v_{\varepsilon}\right)
$$

Recall that the unique solution of (3.9) satisfies $\rho_{\mathrm{TF}}\left(\nabla S_{\varepsilon}-\boldsymbol{\Omega} \times \mathbf{r}\right)=\Omega$ curl $\boldsymbol{\Xi}_{\varepsilon}$. Hence we integrate by part in (3.30) to get

$$
I_{\varepsilon}\left(v_{\varepsilon}\right)=\Omega \int_{\mathcal{D}} \boldsymbol{\Xi}_{\varepsilon} \cdot \operatorname{curl}\left(i v_{\varepsilon}, \nabla v_{\varepsilon}\right)
$$

Let $\phi_{\varepsilon}$ be the phase of $v_{\varepsilon}$. Since $v_{\varepsilon}$ is tending to one everywhere except on the vortex line, then $\left(i v_{\varepsilon}, \nabla v_{\varepsilon}\right) \sim \nabla \phi_{\varepsilon}$, hence we can approximate $\operatorname{curl}\left(i v_{\varepsilon}, \nabla v_{\varepsilon}\right)$ by $2 \pi \vec{\delta}_{\gamma}$. We use the value of $\boldsymbol{\Xi}$ given by (3.11) and the fact that $\dot{\gamma}(t) \cdot \mathbf{e}_{z}=d z$ to get

$$
I_{\varepsilon}\left(v_{\varepsilon}\right) \simeq-\frac{\Omega \pi}{\left(1+\alpha^{2}\right)} \int_{\gamma} \rho_{\mathrm{TF}}^{2} d z
$$

\subsubsection{Final estimate for the energy}

We use (3.13)-(3.19)-(3.29)-(3.31) to derive the energy of a solution with a vortex line $\gamma$. Indeed the energy of any solution minus the energy of a solution without vortex is roughly the vortex contribution in the sense:

$$
E_{\varepsilon}\left(u_{\varepsilon}\right)-E_{\varepsilon}\left(\eta_{\varepsilon}\right) \simeq \mathcal{E}_{\gamma}
$$

We find that the vortex contribution $\mathcal{E}_{\gamma}$ is

$$
\mathcal{E}_{\gamma}=\int_{\gamma} \rho_{\mathrm{TF}}\left(\frac{c_{*}}{2}+\pi \log \left(\frac{2}{\varepsilon e^{C_{0}}} \sqrt{\frac{\rho_{\mathrm{TF}}}{\mu+\frac{k^{2}}{4}}}\right)\right) d l-\frac{\Omega \pi}{\left(1+\alpha^{2}\right)} \int_{\gamma} \rho_{\mathrm{TF}}^{2} d z .
$$

Hence if the right-hand-side of (3.33) is negative, it means that it is energetically favorable to have vortices. Note that in the first integral of $\mathcal{E}_{\gamma}$, we have $d l=|\dot{\gamma}(z)| d z$ whereas in the second one, we have $d z$, that is an oriented integral. This oriented integral precludes the minimal configuration to have closed loops. 


\subsubsection{Case of several vortices}

Let us assume that the solution $u_{\varepsilon}$ has $n$ vortices along the lines $\gamma_{i}, 1 \leq i \leq n$. We want to estimate the energy in this case. For each $\gamma_{i}$, we define $T_{i, \lambda \varepsilon}$ as in (3.17).

One can check that the estimates (3.31) and (3.19), respectively for $I_{\varepsilon}\left(v_{\varepsilon}\right)$ and for $G_{\varepsilon}\left(v_{\varepsilon}\right)$ close to each vortex core, are unchanged if the integral along $\gamma$ is replaced by the sum of the integrals along $\gamma_{i}$. The only difference is for the estimate away from the vortex cores where we have to take into account the interaction between the vortex lines. Let us denote $\mathcal{D}_{n}=\mathcal{D} \backslash \cup_{j} T_{j, \lambda \varepsilon}$. We still have

$$
G_{\varepsilon}\left(v_{\varepsilon}\right)_{\mid \mathcal{D}_{n}} \simeq \int_{\mathcal{D}_{n}} \frac{1}{2 \rho_{\mathrm{TF}}}|\operatorname{curl} \Psi|^{2}
$$

where $\boldsymbol{\Psi}=\sum_{i} \boldsymbol{\Psi}_{i}$ and $\boldsymbol{\Psi}_{i}$ solves (3.21) with $\gamma_{i}$ instead of $\gamma$. Thus, we need to estimate

$$
\sum_{i} \int_{\mathcal{D}_{n}} \frac{1}{2 \rho_{\mathrm{TF}}}\left|\operatorname{curl} \boldsymbol{\Psi}_{i}\right|^{2}+\sum_{i \neq k} \int_{\mathcal{D}_{n}} \frac{1}{2 \rho_{\mathrm{TF}}} \operatorname{curl} \boldsymbol{\Psi}_{k} \cdot \operatorname{curl} \boldsymbol{\Psi}_{i} .
$$

The first integral is estimated as in section C.2 by

$$
\sum_{i}-\pi \int_{\gamma_{i}} \rho_{\mathrm{TF}} \log \left(\frac{e^{C_{0}}}{2} \sqrt{\mu+\frac{k^{2}}{4}} \lambda \varepsilon\right) d l .
$$

As for the second integral in (3.35), we integrate it by part to get

$$
\pi \sum_{i \neq k} \int_{\gamma_{i}} \Psi_{k} \cdot d l
$$

The computation of $\boldsymbol{\Psi}_{k}(x)$ from the previous section is still valid and we have

$$
\mathbf{\Psi}_{k}(x) \simeq-\rho_{\mathrm{TF}} K_{0}\left(\sqrt{\mu+\frac{k^{2}}{4}} \operatorname{dist}\left(x, \gamma_{k}\right)\right) \dot{\gamma}
$$

This yields the contribution of $n$ vortex lines (to be compared with (3.33) for 1 vortex)

$$
\begin{aligned}
\mathcal{E}_{n}= & \sum_{i} \int_{\gamma_{i}} \rho_{\mathrm{TF}}\left(\frac{c_{*}}{2}+\pi \log \left(\frac{2}{\varepsilon e^{C_{0}}} \sqrt{\frac{\rho_{\mathrm{TF}}}{\mu+\frac{k^{2}}{4}}}\right)\right) d l-\frac{\Omega \pi}{\left(1+\alpha^{2}\right)} \int_{\gamma_{i}} \rho_{\mathrm{TF}}^{2} d z \\
& -\pi \sum_{i \neq k} \int_{\gamma_{i}} \rho_{\mathrm{TF}} K_{0}\left(\sqrt{\mu+\frac{k^{2}}{4}} \operatorname{dist}\left(x, \gamma_{k}\right)\right) d l,
\end{aligned}
$$

where $K_{0}$ is a modified Bessel function. The extra term in the energy models the interaction between vortex lines. Note that the curves are going to interact only in the region where they are close to one another. 


\subsection{Vortex bending}

\subsubsection{Numerical results}

If the vortex line is straight, our computation yields

$$
\frac{\rho_{0}^{3 / 2}}{\beta}\left(\frac{2}{3}\left(\frac{c_{*}}{2}+\pi \log \left(\frac{\sqrt{2}}{\varepsilon e^{C_{0}}}\right)\right)+\frac{2 \pi}{3} \log \rho_{0}+\pi\left(\frac{-10}{9}+\frac{4}{3} \log 2\right)-\Omega \frac{8 \pi \rho_{0}}{15\left(1+\alpha^{2}\right)}\right) .
$$

Making this expression equal to 0 allows to derive a critical angular velocity $\Omega_{1}$ for which a straight vortex has a lower energy than a vortex free solution. With our experimental data, it yields $\Omega_{1} \sim 22.45$. We are going to see that there is a range of value of $\Omega$ less than $\Omega_{1}$ for which a bent vortex has a negative energy and in particular a lower energy than a straight vortex.

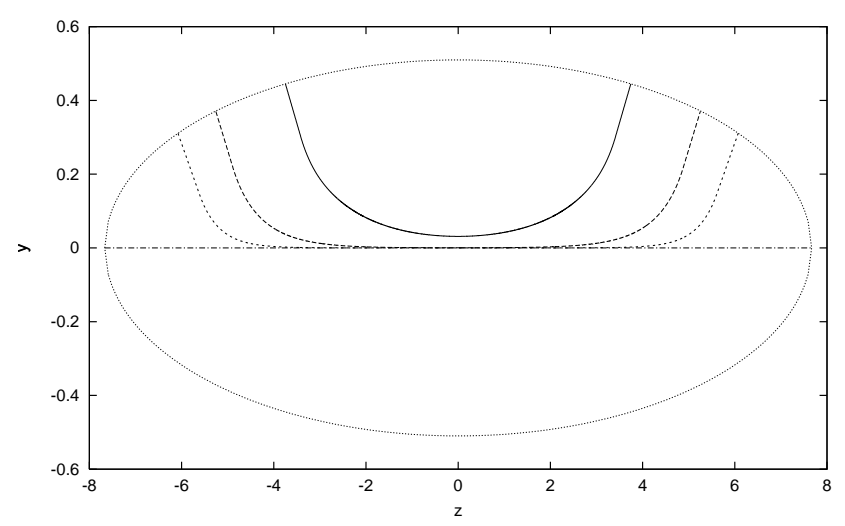

Figure 3.4: The vortex line for various values of $\Omega$ in the $z-y$ plane: $\Omega=21.8$ (straight line), $\Omega=25.8$ (dotted line), $\Omega=33.1$ (dashed line).

We plot the energy of the straight vortex line and the bent vortex vs $\Omega$ in Figure 3.5. One can observe that for $\Omega_{c}=21.8$, in the initial units, the energy of the bent vortex starts to be negative (that is below the energy of a solution without vortex), while the energy of a straight vortex line is positive. For $\Omega=33.1$, the energy of the bent vortex and of a straight vortex line become equal.

Let us point out that the bent vortex is a minimizer even if the cross section is a disc. Nevertheless, when $\varepsilon$ is fixed, if $\beta$ gets too big, the straight vortex becomes the minimizer, which is the case for $\beta=1$. 


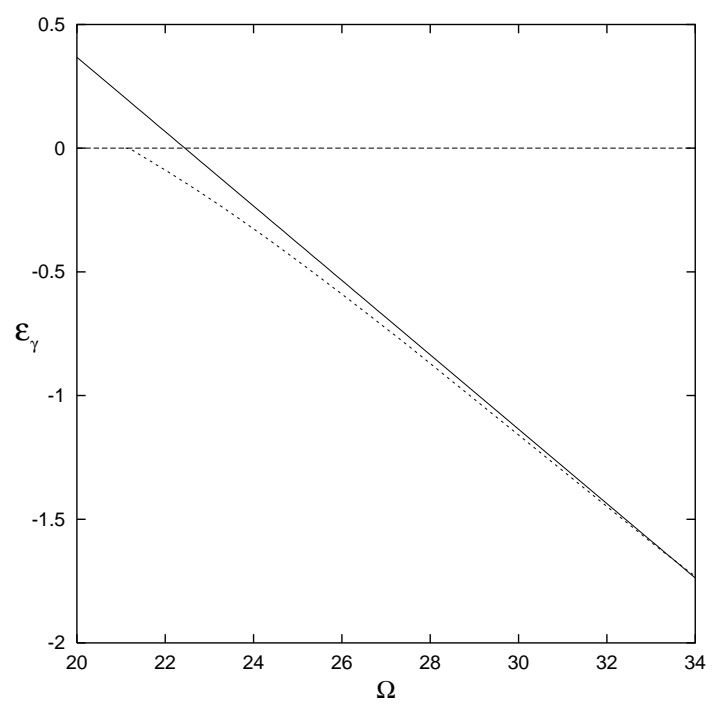

Figure 3.5: The energy vs. $\Omega$ curves for the solution with a straight vortex (solid line) and a bent vortex (dotted line).

\subsubsection{Rigorous results}

For a rigorous study, we are going to make a simplification on the energy $\mathcal{E}_{\gamma}$, valid for small $\varepsilon$. After rescaling the energy and $\Omega$ by some appropriate constant, we are lead to

$$
E[\gamma]=\int_{\gamma} \rho_{\mathrm{TF}} d l-\Omega \int_{\gamma} \rho_{\mathrm{TF}}^{2} d z
$$

The energy $E[\gamma]$ reflects the competition between the vortex energy due to its length (1st term) and the rotation term. Note that the rotation term is an oriented integral ( $d z$ not $d l$ ), which actually forces the vortex to be along the $z$ axis, while the other term wants to minimize the length. This is why, according to the geometry of the trap, the shape of the vortex varies. In this scaling, the energy of the vortex free solution is zero. Thus, a vortex line is energetically favorable when $\Omega, \beta$ are such that $\inf _{\gamma} E[\gamma]<0$.

First of all, it has been observed numerically [GP] that the vortex line lies in the plane closest to the axis of rotation and we can provide a rigorous justification:

Theorem 3.1 If $\alpha \geq 1$, then the energy is minimized when the vortex line lies in the $(y, z)$ plane, that is the plane closest to the axis.

Indeed, if we have a curve $\gamma$ parametrized as $\gamma(t)=(x(t), y(t), z(t))$, then we can define the new curve $\tilde{\gamma}(t)=(0, \tilde{y}(t), \tilde{z}(t))$ by $\tilde{z}(t)=z(t)$ and $\tilde{y}(t)=\sqrt{x^{2}+\alpha^{2} y^{2}}$. Then $\rho_{\mathrm{TF}}(\gamma(t))=$ $\rho_{\mathrm{TF}}(\tilde{\gamma}(t))$. Since $\alpha>1, \dot{\tilde{y}}^{2} \leq \dot{x}^{2}+\dot{y}^{2}$, hence $\rho(\tilde{\gamma})|\dot{\tilde{\gamma}}|-\Omega \rho(\tilde{\gamma}) \dot{\tilde{z}} \leq \rho(\gamma)|\dot{\gamma}|-\Omega \rho(\gamma) \dot{z}$. It follows that the energy of the new curve $E[\tilde{\gamma}]$ is less or equal than $E[\gamma]$. If $\alpha=1$, that is the cross section is a disc, then our arguments imply that the vortex line is planar, but of course all transversal planes are equivalent. 
From now on, we will assume that the curve lies in the plane $(y, z)$, so that $\rho_{\text {TF }}$, only depends on $y$ and $z$ and we will call it $\rho$. Recall from the expression of $E$, (3.40), that for $E[\gamma]$ to be negative, we need $\rho-\Omega \rho^{2}$ to be negative somewhere, that is $\Omega \rho>1$. For fixed $\Omega$, we define the regions

$$
\mathcal{D}_{i}:=\{(y, z): \Omega \rho(y, z)>1\}, \quad \mathcal{D}_{o}:=\mathcal{D} \backslash \mathcal{D}_{i} .
$$

We will refer to these sets as "the inner region" $\mathcal{D}_{i}$ and "the outer region" $\mathcal{D}_{o}$ respectively. In the outer region, the energy of a vortex per unit arc length is necessarily positive, since $\rho-\Omega \rho^{2}>0$, whereas in the inner region, for appropriately oriented vortices it can be negative since $\rho-\Omega \rho^{2}<0$. One can see easily that for $\gamma$ to have a negative energy, part of the vortex line has to lie in the inner region, that is close to the center of the cloud. Note that for $\mathcal{D}_{i}$ to be non empty, we need at least $\Omega \rho_{0}>1$. In the region $\mathcal{D}_{i}$, we will see that the vortex is close to the axis for all $\beta$. On the other hand, in the region $\mathcal{D}_{o}$, the vortex goes to the boundary along the quickest path: if $\beta$ is small, perpendicularly to the boundary, which gives rise to a bent vortex and if $\beta>1$, the vortex stays along the axis of rotation.

Let $\gamma_{s}$ be a straight vortex along the $z$ axis. We say that the straight vortex is stable if $\left(v, E^{\prime \prime}\left[\gamma_{s}\right] v\right)>0$ for all $v$, and unstable if $\left(v, E^{\prime \prime}\left[\gamma_{s}\right] v\right)<0$ for some $v$.

Theorem 3.2 The straight vortex is stable if

$$
\Omega \rho_{0}>\frac{3}{4}+\frac{1}{4 \beta^{2}}
$$

The straight vortex is unstable if $\beta<1 / \sqrt{3}$ and

$$
\Omega \rho_{0}<\frac{1}{6}+\frac{1}{6 \beta^{2}}
$$

Note that the 2 values are consistent in the sense that they both scale like $1 / \beta^{2}$ when $\beta$ is small. For $\Omega$ large, one expects several vortices in the condensate, but the fact that a straight vortex is stable gives an indication that for $\Omega$ large, each vortex should be nearly straight, which is consistent with the observations [AM].

It is interesting to see what happens in Theorem 3.2 when $\Omega \rho_{0}=5 / 4$, that is when the straight vortex has zero energy. The first inequality yields that if $\beta>1 / \sqrt{2}$, then the straight vortex is stable for all $\Omega$ such that $\Omega \rho_{0}>5 / 4$, that is when $E\left[\gamma_{s}\right]<0$. If $\beta>1$, we have that $\gamma_{s}$ is not just stable but in fact minimizes $E$. The second inequality implies that, if $\beta<\sqrt{2 / 13} \approx .39$ then the straight vortex is unstable at the velocity $\Omega \rho_{0}=5 / 4$ at which $E\left[\gamma_{s}\right]=0$. As a result, for these values of $\beta$, the first vortex to nucleate as $\Omega$ increases is a bent vortex. Note that it has been observed in [SvF2] that for $\beta \lesssim 1 / 2$, the ground state of the system exhibits a bent vortex. Numerical results of [GP] also show that bent vortices are energetically favorable when $\beta$ is small.

All this indicates that by varying the elongation of the condensate, one may hope to go from a situation where the first vortex is bent, to a situation where it is straight. 
For a curve $\gamma$, let $I_{\gamma, i}:=\left\{t \in I: \gamma(t) \in \mathcal{D}_{i}\right\}$ and $I_{\gamma, o}=I \backslash I_{\gamma, i}$. And let $\gamma_{i}$ be the restriction of $\gamma(\cdot)$ to $I_{\gamma, i}$, and similarly $\gamma_{o}$.

The definition of $I_{\gamma, o}$ implies that $\rho(\gamma(t))-\Omega \rho^{2}(\gamma(t))>0$ for $t \in I_{\gamma, o}$, and as a consequence

$$
\rho(\gamma(t))|\dot{\gamma}(t)|-\Omega \rho^{2}(\gamma(t)) \dot{z} \geq|\dot{\gamma}(t)|\left(\rho(\gamma(t))-\Omega \rho^{2}(\gamma(t))\right)
$$

which is positive in $I_{\gamma, o}$. Thus if $\gamma$ is such that $I_{\gamma, i}$ is empty, then clearly $E[\gamma]>0$ and it is energetically favorable not to have a vortex. This is the case in particular for $\Omega \rho_{0}<1$ since then $\mathcal{D}_{i}$ is empty. We may thus restrict our attention to the case $I_{\gamma, o}$ nonempty.

Proposition 3.1 For all $\beta$ and all $\Omega$, in the inner region, the straight vortex minimizes the energy, that is $M_{i}=\inf \left\{E\left[\gamma_{i}\right]\right\}$, where $\gamma_{i}$ is the restriction of $\gamma(\cdot)$ to $I_{\gamma, i}$, is attained by the straight vortex.

Proposition 3.2 For $\beta \geq 1$, in the outer region, the straight vortex minimizes the energy, that is the infimum $M_{o}$ of $\left\{E\left[\gamma_{o}\right]\right\}$, where $\gamma_{o}$ is the restriction of $\gamma(\cdot)$ to $I_{\gamma, o}$, is attained by the straight vortex.

Note that in the outer region, Proposition 3.2 only holds for $\beta>1$. If $\beta<1$, the situation is somewhat more complicated: $\int_{\gamma_{o}} \rho d l$ is minimized by a path that joins $\mathcal{D}_{i}$ to $\partial \mathcal{D}$ along the $y$ axis, whereas $-\int_{\gamma_{o}} \rho^{2} d z$ is minimized by the straight vortex running along the $z$-axis. The minimizer of the full energy reflects the competition between these two terms, and hence is bent.

In the case $\beta<1$, that is when the vortex line is bent, we can prove that the vortex has a minimum length. This is related to the fact that the vortex has to go to the center of the cloud and spend some time in the inner region.

For an open set $U \subset \mathcal{D}$ with Lipschitz boundary, we endow $\partial U$ with an orientation in the standard way, so that Stokes' theorem holds.

We will prove the following isoperimetric-type inequality:

Theorem 3.3 For every $0<\beta \leq 1$

$$
\left|\int_{\partial U} \rho^{2} d z\right| \leq\left(2 \sqrt{\rho_{0}}\right)^{1 / 2}\left(\int_{\partial U} \rho d l\right)^{3 / 2}
$$

for every connected open subset $U \subset \mathcal{D}$,

A short calculation starting from (3.45) shows that if $E[\gamma]<0$ then

$$
\int_{\gamma} \rho d l>\frac{1}{\left(2 \Omega^{2} \sqrt{\rho_{0}}\right)}
$$

We expect that even for a configuration with multiple vortices, each vortex line will satisfy a lower bound of the type (3.46). In a configuration with several vortices $\gamma_{k}$, the energy derived in $[\mathrm{AR}]$ is $\sum E\left[\gamma_{k}\right]+I\left(\gamma_{k}, \gamma_{j}\right)$, where

$$
I\left(\gamma_{k}, \gamma_{j}\right)=\int_{\gamma_{k}} \mid \log \left(\operatorname{dist}\left(x, \gamma_{j}\right) \mid d l\right.
$$


Adding a vortex to a stable configuration with $n-1$ vortices, requires

$$
E\left[\gamma_{n}\right]+\sum I\left(\gamma_{n}, \gamma_{j}\right)<0
$$

Since $I>0$, this implies in particular that $E\left[\gamma_{n}\right]<0$ and hence the bound on the length.

\subsection{Conclusion}

We have obtained a simplified expression (3.33) of the energy of a minimizing solution of the Gross Pitaevskii energy with a vortex line $\gamma$ and (3.38) for $n$ vortex lines $\gamma_{i}$. This expression depends on the shape of the vortex line. It has a term coming from the energy of vortices and another one due to the angular momentum of vortices This has allowed us to draw the vortex line for the minimizing solution and compute its energy. We have seen that there is a range of rotational velocities for which a bent vortex line has a lower energy than a straight vortex and a vortex free solution. These computations on the simplified expression of the energy are in agreement with the computations on the full energy [GP]. 


\section{Bibliography}

[A1] A.Aftalion On the minimizers of the Ginzburg-Landau energy for high kappa: the one dimensional case. European Journal of Applied Mathematics, 8 (1997) 331- 345.

[A2] A.Aftalion On the minimizers of the Ginzburg-Landau energy for high kappa: the axially symmetric case. Annales de l'Institut Henri Poincaré (Analyse non linéaire) 16 (1999) $747-772$

[AC1] A.Aftalion \& S.J.Chapman Asymptotic analysis of the bifurcation diagram for symmetric one dimensionnal solutions of the Ginzburg-Landau equations. European Journal of Applied Mathematics 10 (1999) 477-495.

[AC2] A.Aftalion \& S.J.Chapman Asymptotic analysis of a secondary bifurcation of the one dimensionnal Ginzburg-Landau equations of superconductivity. SIAM J. Applied Maths. 60 (2000) 1157-1176.

[AD] A.Aftalion \& E.N.Dancer On the symmetry of solutions of the Ginzburg-Landau equations for small domains. Com. Contemporary Maths. 3 (2001) 1-14.

[ADu1] A.Aftalion \& Q.Du The bifurcation diagrams for the Ginzburg-Landau system of superconductivity. Physica D.

[ADu2] A.Aftalion \& Q.Du Vortices in a rotating Bose-Einstein condensate: critical velocities and bifurcation diagrams in the Thomas-Fermi regime. Phys. Rev A 64 (2001) Vol.6, 063603.

[AJ] A.Aftalion \& R.L.Jerrard Shape of vortices for a rotating Bose Einstein condensate Phys. Rev A (2002).

[ASS] A.Aftalion, E.Sandier \& S.Serfaty Pinning phenomena in the Ginzburg-Landau model of superconductivity. J. Maths Pures Appl. 80 (2001) 339-372.

[AR] A.Aftalion \& T.Riviere Vortex energy and vortex bending for a rotating Bose-Einstein condensate. Phys. Rev A 64 (2001) Vol.4, 043611.

[AT1] A.Aftalion \& W.C.Troy On the solutions of the one-dimensionnal Ginzburg-Landau equations for superconductivity. Physica D 132 (1999) 214-232. 
[AT2] A.Aftalion \& W.C.Troy Uniqueness of solutions of the Ginzburg-Landau system for thin films. European Journal of Applied Mathematics 11 (2000) 365-380.

[AM] E.Akkermans \& K.Mallick Vortices in Ginzburg-Landau billiards. J. Phys. A 32 (1999), no. 41, 7133-7143.

[BPV] B.Baelus, F.M.Peeters \& V.Schweigert Vortex states in superconducting rings, Phy. Rev. B, 61, (2000), 9734-9747.

[BPT] P.Bauman, D.Phillips \& Q.Tang Stable nucleation for the Ginzburg-Landau system with an applied magnetic field. Arch. Rational Mech. Anal. 142 , (1998) no. 1, 1-43.

[BBC] H.Berestycki, A.Bonnet \& S.J.Chapman A semi-elliptic system arising in the theory of superconductivity. Comm. Appl. Nonlinear Anal. 1, (1994) 1-21.

[BS] A.Bernoff \& P.Sternberg, Onset of superconductivity in decreasing fields for general domains. J.Math.Phys. 39 (1998) 1272-1284.

[BBH] F. Bethuel, H. Brezis \& F. Helein, Ginzburg-Landau Vortices, (1994) Birkhäuser.

[BR] F. Bethuel and T. Riviere, Vortices for a Variational Problem Related to Superconductivity, Annales IHP, Analyse non linaire, 12, (1995), 243-303.

[BC] T. Boeck \& S.J.Chapman Bifurcation to vortex solutions in superconducting films. European J. Appl. Math. 8, (1997) 125-148.

[BH1] C.Bolley \& B.Helffer An application of semi-classical analysis to the asymptotic study of the supercooling field of a superconducting material. Ann. Inst. Henri Poincaré (Phys. Théorique) 58, (1993), 189-233.

[BH2] C.Bolley \& B.Helffer Rigorous results on Ginzburg-Landau models in a film submitted to an exterior parallel magnetic field I. Nonlinear Stud. 3, (1996), 1-29.

[BH3] C. Bolley \& B.Helffer Rigorous results on Ginzburg-Landau models in a film submitted to an exterior parallel magnetic field II. Nonlinear Stud. 3, (1996), 121-152.

[BH4] C.Bolley \& B.Helffer Rigorous results for the Ginzburg-Landau equations associated to a superconducting film in the weak $\kappa$ limit. Rev. Math. Phys. 8, (1996), 43-83.

[BH5] C.Bolley \& B.Helffer The Ginzburg-Landau equations in a semi-infinite superconducting film in the large $\kappa$ limit. European J. Appl. Math. 8, (1997), 347-367.

[BH6] C. Bolley \& B.Helffer Stability of bifurcating solutions for the Ginzburg-Landau equations. Rev. Math. Phys. 10 (1998), 579-626.

[BuR] D.Butts \& D.Rokhsar, Nature 397, 327 (1999). 
[Ch1] S.J. Chapman Nucleation of superconductivity in decreasing fields I. Europ. J. Appl. Math. 5, (1994), 449-468.

[Ch2] S.J. Chapman Nucleation of superconductivity in decreasing fields II. Europ. J. Appl. Math. 5, (1994), 469-494.

[CDG] S. J. Chapman, Q. Du, and M.D. Gunzburger, A Ginzburg-Landau type model of superconducting / normal junctions including Josephson junctions, Eur. J. Appl. Math., 6, (1995), 97-114.

[CHMLO] S.J.Chapman, S.D.Howison, J.B.McLeod, \& J.R. Ockendon Normal superconducting transitions in Landau-Ginzburg theory. Proc. Royal Soc. Edinburgh, 119 (1991), 117-124.

[CHO] S.J. Chapman, S.D.Howison \& J.R.Ockendon Macroscopic models of superconductivity. SIAM Review, 34 4, (1992) 529-560.

[CR] S. J. Chapman, and G. Richardson Vortex pinning by inhomogeneities in type II superconductors. Phys. D 108 (1997), no. 4, 397-407.

[CD] Y.Castin \& R.Dum, Eur. Phys. J. D, 7, 399 (1999).

[CD] D. Cioranescu and P. Donato, Introduction to homogenization, Oxford University Press, (1999).

[CR] M.G.Crandall \& P.H.Rabinowitz Bifurcation, perturbation from simple eigenvalues and linearized stability. Arch. Rational Mech. Anal. 52 (1973), 161-180.

[CP] M.Cyrot \& D.Pavuna 1992 Introduction to superconductivity and high- $T_{c}$ materials. World Scientific.

[DGPS] F.Dalfovo, S.Giorgini, L.Pitaevskii \&S.Stringari, Rev. Mod. Phys. 71,463 (1999).

[DFS] M.Del Pino, P.Felmer \& P.Sternberg Boundary concentration for eigenvalue problems related to the onset of superconductivity. Comm. Math. Phys. 210 (2000), no. $2,413-446$.

[DPS] P.Deo, F.M.Peeters \& V.A.Schweigert Mesoscopic superconducting disks. Superlattices and microstructures, 25 (1999), 1195-1211.

[Do1] E.J.Doedel, A.R.Champneys, T.F.Fairgrieve, Y.A.Kuznetsov, B.Sandstede \& X.J.Wang 1997 AUTO97: Continuation and bifurcation software for ordinary differential equations. Available by FTP from ftp.cs.concordia.ca/pub/doedel/auto.

[Do2] E.J.Doedel AUTO: A program for the automatic bifurcation analysis of autonomous systems. Cong. Num. (Proc. 10th Manitoba Conf. on Num. Math. and Comp., Univ. of Manitoba, Winnipeg, Canada) 30, (1981), 265-284. 
[DG] Q.Du \& P.Gray High-kappa limit of the time dependent Ginzburg-Landau model for superconductivity, SIAM J. Appl. Math., 56, (1996), pp1060-1093.

[DGP1] Q. Du, M.D. Gunzburger and J.S. Peterson Analysis and approximation of the Ginzburg-Landau model of superconductivity. SIAM Review, 34, 1, (1992), 54-81.

[DGP] Q. Du, M.D. Gunzburger and J.S. Peterson, Computational simulations of type II superconductivity including pinning phenomena, Ph. Rev. B, 51, N. 22, (1995) 16194-16203.

[F] L.E. Fraenkel An introduction to maximum principles and symmetry in elliptic problems. Cambridge tracts in mathematics 128, Cambridge University Press, (1997).

[FPR] T.Frisch, Y.Pomeau \& S.Rica Transition to dissipation in a model of superflow (1992) Phys. Rev. Let. 691644.

[GP] J.J.García-Ripoll and V.M.Perez-García, cond-mat/0102129.

[G] P.G. de Gennes 1966 Superconductivity of metals and alloys. Addison Wesley.

[GNN] B.Gidas, W.M.Ni \& L.Nirenberg Symmetry and related properties via the maximum principle. Comm. Math. Phys. 68 (1979), 209-243.

[GS] B.Gidas \& J.Spruck Global and local behavior of positive solutions of nonlinear elliptic equations. Comm. Pure Appl. Math. 34 (1981), 525-598.

[GT] D. Gilbarg \& N.S. Trudinger Elliptic partial differential equations of second order. 2nd edition, Springer Verlag, 1983.

[G] V.L.Ginzburg On the destruction and onset of superconductivity in a magnetic field. Soviet Phys. JETP, 34, (1958) 78-87.

[GL] V.L.Ginzburg \& L.D.Landau On the theory of superconductivity. Soviet Phys. JETP, 20, (1950), 1064-1082. English translation: Men of physics: L.D.Landau ed. D.ter Harr, Pergamon, Oxford (1965) 138-167.

[GP] T.Giorgi \& D.Phillips The breakdown of superconductivity due to strong fields for the Ginzburg-Landau model. SIAM J. Math. Anal. 30, (1999), 341-359.

[GS] S.Gueron \& I.Shafrir On a discrete variational problem involving interacting particles. SIAM J. Appl. Math. 60 (2000), no. 1, 1-17.

[HLM] V.Hakim, A.Lemaitre \& K.Mallick Giant vortices in the Ginzburg-Landau description of superconductivity. (2001) preprint.

[HKT] S.P.Hastings, M.K.Kwong \& W.C.Troy The existence of multiple solutions for a Ginzburg-Landau type model of superconductivity. Europ. J. Appl. Math. 7, (1996), 559-574. 
[HT] S.P.Hastings \& W.C.Troy There are asymmetric minimizers for the one dimensionnal Ginzburg-Landau model of superconductivity. SIAM J. Math. Anal. 30 (1999), no. 1, $1-18$.

[HM] B.Helffer \& A.Morame Magnetic bottles in connection with with superconductivity. Preprint (2000).

[HP] B.Helffer \& X.B.Pan Upper critical field and location of surface nucleation of superconductivity. Preprint (2001).

[J] H.T.Jadallah The onset of superconductivity in a domain with corner. (2000) Preprint.

[Kw] M.K. Kwong On the one-dimensional Ginzburg-Landau BVPs. Diff. Int. Equations 8, (1995), 1395-1405.

[KwZ] M.K.Kwong \& L.Q.Zhang Uniqueness of the positive solution of $\Delta u+f(u)=0$ in an annulus. Differential Integral Equations 4 (1991), no. 3, 583-599.

[LM] L.Lassoued \& P.Mironescu Ginzburg-Landau type energy with discontinuous constraint. J. Anal. Math. 77 (1999), 1.

[L] K. Likharev, Superconducting weak links, Rev. Mod. Phys., 51, (1979), 101-159.

[LD] F.H.Lin \& Q.Du Ginzburg-Landau vortices: dynamics, pinning and hysteresis, SIAM J. Math. Anal., 28, (1997) pp.1265-1293.

[LP1] K.Lu \& X.Pan Estimates of the upper critical field for the Ginzburg-Landau equations of superconductivity. Phys. D 127 (1999), no. 1-2, 73-104.

[LP2] K.Lu \& X.Pan Eigenvalue problems of Ginzburg-Landau operator in bounded domains. J. Math. Phys. 40 (1999), no. 6, 2647-2670.

[LP3] K.Lu \& X.Pan Surface nucleation of superconductivity in 3-dimensions. Special issue in celebration of Jack K. Hale's 70th birthday, Part 2 (Atlanta, GA/Lisbon, 1998). J. Differential Equations 168 (2000), no. 2, 386-452.

[MCBD] K. Madison, F. Chevy, V. Bretin \& J. Dalibard, Phys. Rev. Lett., 84, 806 (2000).

[MCDW] K. Madison, F. Chevy, W. Wohlleben \& J. Dalibard, J.Mod.Opt., 47, 2715 (2000).

[MK] M.H.Millman \& J.B.Keller 1969 Perturbation Theory of Nonlinear Boundary-Value Problems. J. Math. Phys. 10, 2, 342.

[OK] R.Onofrio, C.Raman, J.M.Vogels, J.Abo-Shaeer, A.P.Chikkatur \& W.Ketterle. Phys. Rev. Lett., 85, 2228 (2000). 
[PR] F.Pacard \& T. Riviere Linear and nonlinear aspects of vortices. The GinzburgLandau model. Progress in Nonlinear Differential Equations and their Applications, 39. Birkhuser Boston, Inc., Boston, MA, 2000.

[OS] T.Ouyang \& J.Shi Exact multiplicity of positive solutions for a class of semilinear problems IE II J. Differential Equations 146 (1998), no. 1, 121-156 et 158 (1999), no. $1,94-151$.

[P] X.Pan Surface superconductivity in applied magnetic field above $H_{c_{2}}$. Preprint (2001).

[Pi] L.M. Pismen, Vortices in Nonlinear Fields, International series of monographs on physics, 100, Oxford Science Publications, (1999).

[Rab] P.H.Rabinowitz Pairs of positive solutions of nonlinear elliptic partial differential equations, Indiana Univ. Math. J. 23 (1974), 173-186.

[Ri] T.Riviere, Line vortices in the U(1)-Higgs model. ESAIM Contrle Optim. Calc. Var. 11 77-167 (1996).

[SJdG] D.Saint-James \& P.G.De Gennes Onset of superconductivity in decreasing fields. Phys. Lett. 7 (1963) 306-307.

[SJST] D.Saint-James, G.Sarma \& E.J.Thomas 1969 Type II superconductivity. Pergamon, Oxford.

[SS1] E. Sandier \& S. Serfaty, Global Minimizers for the Ginzburg-Landau Functional below the First Critical Magnetic Field, Annales IHP, Analyse non linéaire 17, 1, (2000), 119-145.

[SS2] E. Sandier \& S. Serfaty On the Energy of Type-II Superconductors in the Mixed Phase, Rev. Math. Phys. 12 (2000), no. 9, 1219-1257.

[SS3] E. Sandier \& S. Serfaty A Rigorous Derivation of a Free-Boundary Problem Arising in Superconductivity, Ann. Sci. cole Norm. Sup. (4) 33 (2000), no. 4, 561-592.

[Ser1] S. Serfaty, Local Minimizers for the Ginzburg-Landau Energy near Critical Magnetic Field Comm. Contemporary Mathematics, 1, No. 2, (1999), 213-254 et 295-333.

[Ser2] S. Serfaty, Stable Configurations in Superconductivity: Uniqueness, Multiplicity and Vortex-Nucleation, Arch. for Rat. Mech. Anal., 149, No 4, (1999), 329-365.

[Ser3] S.Serfaty On a model of rotating superfluids. ESAIM Control Optim. Calc. Var. 6 (2001), 201-238.

[SP] V.A.Schweigert \& F.M.Peeters Phase transitions in thin mesoscopic superconducting disks. Phys. Rev. B, 57, (1998), 13817-13832. 
[SPD] V.A.Schweigert,F.M.Peeters \& P.S.Deo Vortex phase diagram for mesoscopic superconducting disks. Phys. Rev. Lett., 81, (1998), 2783-2786.

[Sey] R.Seydel From equilibrium to chaos; Practical bifurcation and stability analysis. (1988), Elsevier Publ. Co.

[SvF2] A.A.Svidzinsky \& A.L.Fetter, Phys. Rev. A, 62, 63617 (2000).

[TT] D.R.Tilley \& J.Tilley Superfluidity and superconductivity. Adam Hilger, Bristol and New York 1970.

[T] M. Tinkham, Introduction to Superconductivity, 2d edition, McGraw-Hill, (1996). 